\title{
Elastic models of dislocations based on atomistic Kanzaki forces
}

\author{
B. Gurrutxaga-Lerma ${ }^{1,2, *}$ and J. Verschueren ${ }^{3}$ \\ ${ }^{1}$ Trinity College, University of Cambridge, CB2 1TQ Cambridge, United Kingdom \\ ${ }^{2}$ Department of Engineering, University of Cambridge, CB2 1PZ Cambridge, United Kingdom \\ ${ }^{3}$ Department of Materials, Imperial College London, SW7 2AZ London, United Kingdom
}

(Received 23 March 2018; revised manuscript received 10 September 2018; published 11 October 2018)

\begin{abstract}
This paper studies the relationship between the atomistic representation of crystalline dislocations as Kanzaki forces and the continuum representation of dislocations as Burridge-Knopoff (BK) force distributions. We first derive a complete theory of the BK force representation of dislocations in an anisotropic linear elastic continuum, showcasing a number of fundamental features found when dislocations are represented as distributions of body forces in defect-free continuum media. We then build, within the harmonic approximation, the Kanzaki force representation of dislocations in atomistic lattice models. We rigorously show that in the long-wave limit, the Kanzaki force representation converges to the continuum BK representation. We therefore justify employing the Kanzaki forces as source terms in continuum theories of dislocations. We do this by establishing a methodology to compute the Kanzaki forces of dislocations via the force constant matrix of the material's perfect lattice. We use it to study a model of a screw dislocation in bcc tungsten, where we show the existence of two distinct Kanzaki force terms: the slip Kanzaki forces, which we show directly correspond with the BK forces implied by a Volterra dislocation; and the core Kanzaki forces, which are computed from the relaxed dislocation structure, and serve to model all core effects not captured by the Volterra dislocation. We build a multipolar field expansion of both the core and the slip Kanzaki forces, showing that the dislocation core is agreeable to correction via the multipolar field expansion of the core Kanzaki forces.
\end{abstract}

DOI: 10.1103/PhysRevB.98.134104

\section{INTRODUCTION}

Dislocations are crystalline defects defined by an atomic disregistry: the atoms at either side of the slip surface [1] lie misaligned with respect to their equilibrium (perfect lattice) positions. The disregistry is measured with a certain relative vector $\boldsymbol{B}$ known as the Burgers vector; the boundary of the slip surface defines the dislocation line.

Two general approaches may be employed in the study of dislocations in solids, depending on the length scales and timescales of interest. On the one hand, dislocations are inherently atomistic in nature, so one may wish to study the near field about their core via atomistic simulations. Such features influence their mobility, including the crystallographic directions they may glide along [2-4] and the stress levels required to do so [5,6]; they also determine the role temperature [7] and strain rate $[8,9]$ have, amongst many other features. On the other hand, when such detailed studies are less important than the dislocation's effect in the far field, dislocations may be treated as Volterra discontinuities in an elastic continuum, enabling studies of their long-range effects (as may be done using discrete dislocation dynamics [10]). The physical description of the generation and motion of dislocations in

\footnotetext{
*bg374@cam.ac.uk
}

Published by the American Physical Society under the terms of the Creative Commons Attribution 4.0 International license. Further distribution of this work must maintain attribution to the author(s) and the published article's title, journal citation, and DOI. this case would be informed by the atomistic length scales, but their collective behavior leading to macroscopic plasticity arises from the elastic continuum alone. Further homogenizations of dislocations as continuum defects are possible (see for instance [11]), leading to the macroscopic theories of plasticity in the continuum [12].

This paper concerns the physical and mathematical correspondence between dislocations as atomistic, lattice, defects, and as continuum discontinuities. As continuum defects, dislocations are commonly modeled, within the linear elasticity framework, either as displacement discontinuities of the Volterra kind [1,13], or as eigenstrains [14-17], whereby the dislocation is represented as a source of "stress-free" strain. Eigenstrain theory is one of four generally agreed upon ways of describing an internal source in an elastic continuum body, which lead to largely equivalent [18] accounts of defects in solids: (1) as an eigenstrain [15], transformation strain [17], or stress-free strain [14]; (2) as an eigenstress [15] or stress glut [18,19]; (3) as a distributed force representation [20]; (4) as a (seismic) moment tensor [18,21].

As atomistic defects, dislocations may be introduced in a defect-free lattice by imposing the atomic displacements on it necessary to generate the topology of a dislocation. This is a common strategy in the atomistic modeling of dislocations in molecular dynamics (MD) [10,22-24] and density functional theory (DFT) [25-27]. Upon performing a structural minimization, one eventually reaches the predicted atomistic topology of the dislocation [10].

The atomic displacements necessary to generate the topology of a dislocation in an otherwise perfect lattice entail a set 
of reciprocal forces in the perfect lattice, namely, those that would have to be applied on the perfect lattice to generate the dislocation. That is to say, the dislocation is a topological defect characterized by the relative motion of atoms across the slip surface; as such, it occurs in a defective, nonperfect crystalline lattice (the defect being the dislocation), and it carries a long-range elastic field and a certain, finite, elastic energy. However, one may model the dislocation in a perfect lattice instead, and attain the same elastic fields and elastic energy; this is achieved by applying a set of fictitious forces on the atoms, the magnitude and distribution of which is such that it ensures that the elastic fields due to these forces match one to one those of the dislocation qua topological defect. These forces are the so-called Kanzaki forces [28], and are commonly employed in the modeling of point defects in solids [29-31], where they originate [28]. The Kanzaki forces of dislocations have been employed in the past as source terms in lattice dynamics [15,32-43], where they are obtained as the set of forces that, upon being applied on a perfect lattice, would generate the topology of a Volterra dislocation. However, due to the complicated topology of dislocations, their use in studying these extended defects has generally been limited.

This paper is concerned with how, using the atomistic structure of a fully relaxed dislocation, one can build the continuum level model based on the atomistic Kanzaki forces. A force representation is used because atomistic information regarding the dislocation's structure is often available in terms of the atomic displacements off the perfect lattice position; given a displacement field, the force representation of the defect is guaranteed to be unique and exact in both the continuum [18,19] and atomistic systems [15]. The Kanzaki forces of point defects are commonly employed as source terms in the elastic continuum because these forces are nearly linear elastic. In this paper, we show that this is also the case for the Kanzaki forces of dislocations. In order to do so, we exploit the Burridge-Knopoff (1964) theorem [20], which enables representing any elastic source with an equivalent distribution of body forces applied on an undisturbed solid. This stands in direct analogy to the concept of Kanzaki forces, as those are also applied on the defect-free solid (the perfect lattice) to generate the defect. One of the results presented in this paper is precisely that the Burridge-Knopoff forces are the continuum limit of the Kanzaki forces.

By establishing the equivalence between the Kanzaki and Burridge-Knopoff forces, one may produce continuum models of dislocations built solely from atomistic principles. Such models would encompass the computation of the Kanzaki forces necessary to generate a Volterra dislocation, and of the Kanzaki forces necessary to generate the dislocation core. Given that the Kanzaki forces are defined in the harmonic approximation [28,31], it is possible to study both effects separately. In doing so, we shall discuss how to build atomistically informed models of the dislocation core, and what these entail. This introduces a way to account for atomistic core effects in a range of applications [24,44-47] where these are relevant. This will be done by formulating the multipolar field expansion of a dislocation and its core [48], which enable us to subsume the dislocation (and the dislocation core) into a force dipole, a force quadrupole, etc., applied on a single point.

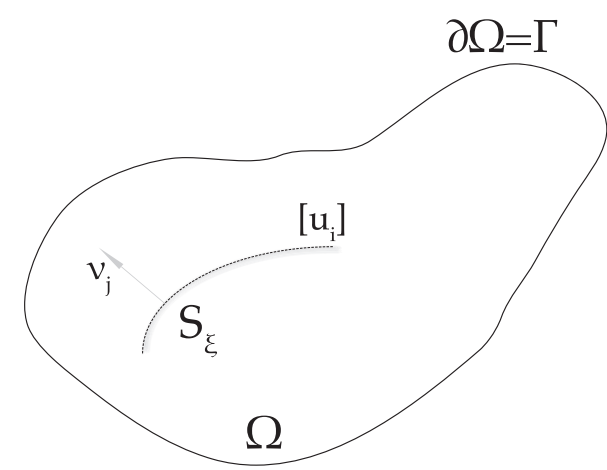

FIG. 1. General configuration of an internal discontinuity $\left[u_{i}\right]$ within a certain elastic body $\Omega$.

The paper is structured as follows. Section II enunciates the Burridge-Knopoff force representation theorem without proof, and goes on to detail what the force representation of dislocations looks like in the continuum. Several examples are provided, which will then be employed in developing the connection between the Burridge-Knopoff and the Kanzaki forces. The latter is done in Sec. III, where, using the harmonic lattice formalism, we derive the atomistic equivalent to the force representation of a dislocation, i.e., the Kanzaki forces, and mathematically prove they converge to the continuum force representation of dislocations in the long-wave approximation. Section IV goes on to compute the Kanzaki forces of a screw dislocation in bcc W. These are then employed in Sec. V to produce an elastic model of the dislocation. Section VI goes on to produce a correction of the dislocation core invoking the multipolar moments of a dislocation, which are explored in detail. Section VII summarizes the main findings of the paper.

\section{DISLOCATIONS AS FORCE DISTRIBUTIONS IN THE ELASTIC CONTINUUM}

\section{A. Burridge-Knopoff theorem}

The Burridge and Knopoff force representation theorem [20] (hereafter, BK theorem) states that any internal discontinuity in the displacement or traction fields of a linear elastic solid may be represented by an equivalent body force distribution applied on a defect-free continuum, such that the elastic fields of the discontinuity and its force equivalent are the same. Here, we state it without proof.

Let $\left[u_{i}\right](\xi)$ be a general displacement discontinuity such as the one represented in Fig. 1. Here, $\boldsymbol{u} \equiv u_{i}$ denotes the displacement field, which is generally dependent on time $t \in \mathbb{R}$ and on a spatial position vector $\xi \in \mathbb{R}^{3}$. Let $S_{\xi}$ be the internal surface defined by the support of $\left[u_{i}\right](\xi)$; let $\boldsymbol{v} \equiv v_{j}$ be the normal vector to said surface. The BK theorem states that the elastic fields due to $\left[u_{i}\right](\xi)$ are equivalent to those due to some force distribution $\boldsymbol{f} \equiv f_{p}$, given by

$$
f_{p}(\boldsymbol{x})=-\int_{S_{\xi}}\left[u_{i}\right](\boldsymbol{\xi}) v_{j} C_{i j p q} \frac{\partial \delta(\boldsymbol{x}-\boldsymbol{\xi})}{\partial x_{q}} d S_{\xi},
$$

where $C_{i j p q}$ is the elastic constant tensor, $\delta(\cdot)$ is Dirac's delta function. For brevity, hereafter

$$
\frac{\partial \delta(\boldsymbol{x}-\boldsymbol{\xi})}{\partial x_{q}}=\delta_{q}(\boldsymbol{x}-\boldsymbol{\xi})
$$


The BK theorem is usually said to entail [18-20] a number of corollaries, two of which will be mentioned in the following: the force representation is such that force and moment equilibrium are guaranteed [20]. As will be discussed in Sec. II B 1 , this is only so long as $\left[u_{i}\right]$ is compactly supported.

\section{B. Volterra dislocations as force distributions}

Knowledge of a $f_{p}(\boldsymbol{x})$ force distribution provides the associated displacement field in an infinite body via the representation theorem [15]

$$
u_{k}(\boldsymbol{x})=\int_{\Omega^{\prime}} G_{k p}\left(\boldsymbol{x}-\boldsymbol{x}^{\prime}\right) f_{p}\left(\boldsymbol{x}^{\prime}\right) d \boldsymbol{x}^{\prime},
$$

where $G_{k p}(\boldsymbol{x})$ is the elastic Green's tensor [15]. Let us consider a closed dislocation loop defined by some closed curve $C$ which represents the dislocation line. The conserved Burgers vector [1] is denoted by $\boldsymbol{B} \equiv B_{i}$. Let $D \in \mathbb{R}^{3}$ be the set of all points enclosed by $C$, i.e., the slip surface. We define the characteristic function $\chi_{D}(\boldsymbol{x})$ associated with the loop as (see [49])

$$
\chi_{D}(x, t)= \begin{cases}0, & x \notin D, \\ 1, & x \in D .\end{cases}
$$

We note that $\iota_{D}(\boldsymbol{x})=-\boldsymbol{v} \cdot \nabla \chi_{D}(\boldsymbol{x})$ is the (compact) surface delta function [50]. The discontinuity dislocation loop may then be modeled as

$$
\left[u_{i}\right](\xi)=B_{i} \chi_{D}(\xi) .
$$

The corresponding force representation of the loop may then be found invoking the BK theorem as

$$
\begin{aligned}
f_{p}(\boldsymbol{x}) & =-\int_{D}\left[u_{i}\right](\boldsymbol{x}) v_{j} C_{i j p q} \delta_{q^{\prime}}\left(\boldsymbol{x}^{\prime}-\boldsymbol{x}\right) d D \\
& =C_{i j p q} B_{i} \frac{\partial}{\partial x_{q}} \int_{D} v_{j} \chi_{D}\left(\boldsymbol{x}^{\prime}\right) \delta\left(\boldsymbol{x}^{\prime}-\boldsymbol{x}\right) d D \\
& =-C_{i j p q} B_{i} v_{j} \frac{\partial \chi_{D}(\boldsymbol{x})}{\partial x_{q}} .
\end{aligned}
$$

\section{Straight dislocations}

Straight dislocations may be regarded as a degenerate case of the dislocation loop, where the $\chi_{D}(\boldsymbol{x})$ represents the positive (or negative) abscissae in the plane.

a. Screw dislocation. We model the screw dislocation in the $(x, y) \equiv\left(x_{1}, x_{2}\right)$ plane in antiplane shear, as a Volterra dislocation satisfying the disregistry

$$
\left[u_{3}\right]\left(x_{1}, x_{2}\right)=B \mathrm{H}\left(x_{1}\right) \delta\left(x_{2}\right),
$$

where $B=|\boldsymbol{B}|$ is the magnitude of the Burgers vector, $\mathrm{H}(\cdot)$ is the Heaviside step function, $u_{z} \equiv u_{3}$ is the antiplane displacement field component; thus, here $\chi_{D}(\boldsymbol{x})=\mathrm{H}\left(x_{1}\right) \delta\left(x_{2}\right)$. The corresponding force representation may be then found invoking Eq. (6):

$$
f_{3}\left(x_{1}, x_{2}\right)=B \mu \mathrm{H}\left(x_{1}\right) \delta^{\prime}\left(x_{2}\right), \quad f_{1}=0=f_{2} .
$$

The force representation of a screw dislocation is shown in Fig. 2(a). As deduced from $f_{3}$ in Eq. (8), the force equivalent of a screw dislocation is a set of force doublets uniformly distributed along the cut surface. The physical meaning of these doublets appears immediate: upon performing the cut along the $x_{3} \equiv z$ direction so as to inject the screw dislocation [see Fig. 2(a)], the cut can only be sustained if a set of equal and opposing forces act along the $z$ direction across the cut surface, so as to maintain the required displacement and, indeed, inject the dislocation.

b. Edge dislocation. The edge dislocation is modeled in the $(x, y) \equiv\left(x_{1}, x_{2}\right)$ plane under plane strain conditions. Modeling the dislocation as a Volterra discontinuity renders

$$
\left[u_{1}\right]\left(x_{1}, x_{2}\right)=B \mathrm{H}\left(x_{1}\right) \delta\left(x_{2}\right) \text {. }
$$

Invoking Eq. (6) with $v_{j}=\left[\begin{array}{lll}0 & 1 & 0\end{array}\right]^{T}$ for $\chi_{D}(\boldsymbol{x})=\mathrm{H}\left(x_{1}\right) \delta\left(x_{2}\right)$, we find

$$
\begin{aligned}
& f_{1}\left(x_{1}, x_{2}\right)=B \mu \mathrm{H}\left(x_{1}\right) \delta^{\prime}\left(x_{2}\right), \\
& f_{2}\left(x_{1}, x_{2}\right)=B \mu \delta\left(x_{1}\right) \delta\left(x_{2}\right), \\
& f_{3}\left(x_{1}, x_{2}\right)=0 .
\end{aligned}
$$

Thus, the force representation of the edge dislocation [Eq. (10)] consists of two components: a distribution of force doublets $f_{1}$ acting along the slip surface, and a force singleton $f_{2}$ perpendicular to the slip surface and acting on the dislocation line. The distribution of force doublets is similar to that of the screw dislocations: it consists of forces of equal magnitude and opposite sign acting across the cut surface in the $x$ direction. However, unlike the screw dislocation, there exists a force singleton $f_{2}$ acting in the $y$ direction. That is, individual dislocations appear to entail force (and torque) imbalances in the body. Similar remarks may be found, without proof, in Nabarro [51] and Kocks and Scattergood [52] in the context of individual dislocations in finite bodies.

\section{Force and moment equilibrium and topological closure in BK force representations of dislocations}

The additional BK force $f_{2}$ in straight edge dislocations arises as a result of the lack of topological closure of the straight dislocation. Indeed, in the following we prove that the support of $\chi_{D}(\boldsymbol{x})$ has to be finite (i.e., that the dislocation's slip surface has to be finite) for the BK theorem to guarantee that the force representation is balanced in force. The total force is

$$
\begin{aligned}
F_{p}^{\mathrm{tot}} & =-\int_{\Omega} C_{i j p q} B_{i} v_{j} \partial_{q} \chi_{D}(\boldsymbol{x}) d \Omega \\
& =-\left.C_{i j p q} B_{i} v_{j} \chi_{D}\left(x_{q}\right)\right|_{-\infty} ^{\infty}+C_{i j p q} B_{i} v_{j} \partial_{q} \int_{D} d D \\
& =0 \Longleftrightarrow D \text { is finite. }
\end{aligned}
$$

Otherwise, the first term does not generally vanish, and therefore $F_{p}^{\text {tot }} \neq 0$ necessarily. A similar argument can be produced for the total torque, which only vanishes if strict topological closure of the defect is enforced:

$$
\begin{aligned}
M_{m}^{\mathrm{tot}} & =-\int_{\Omega} \epsilon_{m n p} C_{i j p q} x_{n} B_{i} v_{j} \partial_{q} \chi_{D}(\boldsymbol{x}) d \Omega \\
& =-\int_{\Omega} \epsilon_{m n p} C_{i j p q} \delta_{q n} B_{i} v_{j} \chi_{D}(\boldsymbol{x}) d \Omega \\
& =-\int_{\Omega} \epsilon_{m q p} C_{i j p q} B_{i} v_{j} \chi_{D}(\boldsymbol{x}) d \Omega=0 \Longleftrightarrow D \text { is finite. }
\end{aligned}
$$




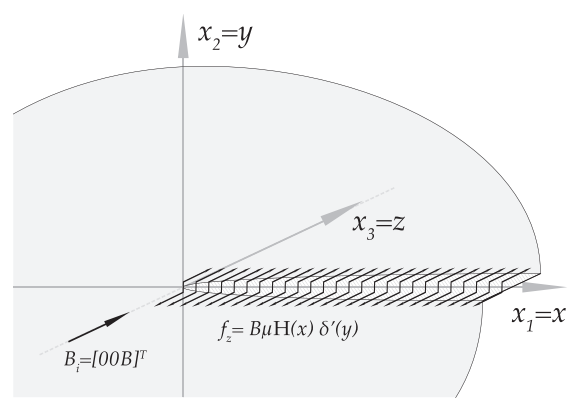

(a) Straight screw dislocation

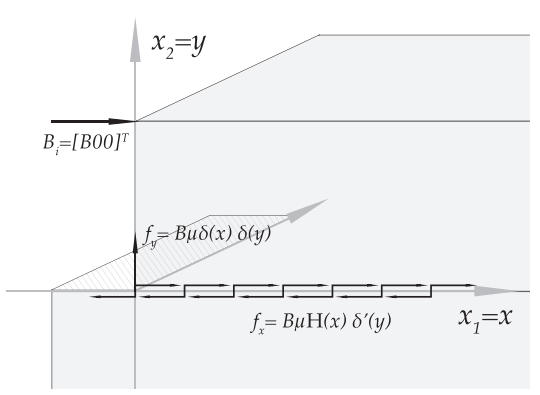

(b) Straight edge dislocation

FIG. 2. Force representation of straight screw and edge dislocations.

This shows that the displacement discontinuity ought to be compactly supported in $\mathbb{R}^{3}$ for there to be force and moment equilibrium.

In both the edge and screw dislocation's case, the total torque is nonzero. Furthermore, in the case of straight edge dislocations, this topological need for closure of the slip surface leads to the appearance of a net nonzero $F_{2}^{\text {tot }}$ component:

$$
\begin{aligned}
F_{2}^{\text {tot }} & =\int_{\mathbb{R} \times \mathbb{R}} f_{2} d x_{1} d x_{2}=B \mu \int_{\mathbb{R} \times \mathbb{R}} \delta\left(x_{1}\right) \delta\left(x_{2}\right) d x_{1} d x_{2} \\
& =B \mu \neq 0,
\end{aligned}
$$

which might be canceled by enforcing local mass conservation, i.e., by considering an edge dislocation dipole of separated by a certain distance $a$. The displacement discontinuity in that case is

$$
\left[u_{1}\right]=B\left[\mathrm{H}\left(x_{1}\right)-\mathrm{H}\left(x_{1}-a\right)\right] \delta\left(x_{2}\right) .
$$

In this case, the force distribution implied by the BK theorem is

$$
\begin{aligned}
& f_{1}=B \mu\left[\mathrm{H}\left(x_{1}\right)-\mathrm{H}\left(x_{1}-a\right)\right] \delta^{\prime}(y), \\
& f_{2}=B \mu\left[\delta\left(x_{1}\right)-\delta\left(x_{1}-a\right)\right] \delta\left(x_{2}\right), \quad f_{3}=0 .
\end{aligned}
$$

Clearly, in this case the $f_{2}$ component is balanced. One may in fact recover Eq. (10) by taking one of the dislocations in the dipole towards infinity, e.g., by taking the $a \rightarrow \infty$ limit. Similarly, the need to enforce moment equilibrium can be shown to lead to requiring dislocation quadrupole clusters in the plane or, otherwise, to considering full dislocation loops, which are the only ones guaranteed to satisfy force and moment equilibrium. We note that this mechanical imbalance does not entail a divergent elastic energy: albeit the individual edge and screw dislocations are shown to transfer a net nonzero torque and force across the body, the elastic energy associated with this deformation is finite, namely, that of the individual Volterra dislocations (see [1]).

\section{DISLOCATIONS AS FORCE DISTRIBUTIONS IN ATOMISTIC MODELS OF A CRYSTALLINE LATTICE}

The continuum force representation of a force loop [Eq. (6)] shows that any dislocation loop (and, by degeneracy, any straight dislocation) can be represented as a force distribution of the form

$$
f_{p}(\boldsymbol{x})=-C_{i j p q} B_{i} v_{j} \partial_{q} \chi_{D}(\boldsymbol{x}) .
$$

This force representation is applied on an "unfaulted" continuum medium [20], and is necessary for producing the topological displacement discontinuity that makes up the Volterra dislocation.

Thus, the BK forces stand in direct analogy to the Kanzaki forces of defects: the Kanzaki forces are the set of body forces that must be applied on a perfect crystalline lattice to induce the displacements that reproduce the topology of a defect [28]. In the case of dislocations, the topology of the defect is the quantized disregistry across the slip surface, the magnitude of which is measured by the Burgers vector [1].

In this section, we examine how such disregistry may be studied from an atomistic perspective, so as to deduce an atomistic equivalent to Eq. (6) within the harmonic lattice formalism. The harmonic lattice formalism is chosen with the aim of recovering Eq. (6) in the long-wave limit. Thus, we shall show that the Burridge-Knopoff forces are the continuum version of the Kanzaki forces.

\section{A. Harmonic lattice formalism}

In the following, we adapt the notation used by Maradudin [53] and Born and Huang [54]. Let us therefore consider a three-dimensional crystal formed by an infinite number of primitive unit cells. The crystal will be assumed to be in static equilibrium. We shall label each unit cell via a vector $\boldsymbol{l}=\left(l_{1} l_{2} l_{3}\right)^{T} \equiv l_{i}$, with $l_{i} \in \mathbb{Z}$, relative to the origin located at $\boldsymbol{l}=\left(\begin{array}{lll}0 & 0 & 0\end{array}\right)^{T}$. The position of each atom in the unit cell relative to the origin of the cell, located at $\boldsymbol{R}(l)=l_{1} \boldsymbol{a}_{1}+l_{2} \boldsymbol{a}_{2}+l_{3} \boldsymbol{a}_{3}$, will be labeled via a certain position vector $\boldsymbol{R}(k)$, so that the global position of the $k$ th atom in cell $l$ may be written as

$$
\boldsymbol{R}(l, k)=\boldsymbol{R}(l)+\boldsymbol{R}(k) .
$$

We denote the relative displacement the $k$ th atom in cell $l$ experiences with respect to its equilibrium position $\boldsymbol{R}(l, k)$ as $\boldsymbol{u}(l, k)=\left(u_{1}(l, k), u_{2}(l, k), u_{3}(l, k)\right)^{T}$. The instantaneous position of the $k$ th atom in cell $l$ will then be given by

$$
\boldsymbol{r}(l, k)=\boldsymbol{R}(l, k)+\boldsymbol{u}(l, k) .
$$

The total potential energy of the lattice $V$ will be assumed to be a function of the instantaneous position of every atom. The interatomic interactions may be described by some potential $\Phi=\Phi(\boldsymbol{r}(l, k))$, which reflects the contribution of the atom $(l, k)$ to the total potential energy [54], so that

$$
V=\sum_{l, k} \Phi(\boldsymbol{r}(l, k)),
$$

is the total potential energy of the crystal. 
Assuming that atomic displacements off equilibrium are small, we expand $V$ about equilibrium in terms of $\boldsymbol{u}(l, k)$ :

$$
\begin{aligned}
V= & V_{0}+\left.\sum_{l, k} \frac{\partial \Phi}{\partial r_{i}(l, k)}\right|_{0} u_{i}(l, k) \\
& +\left.\frac{1}{2 !} \sum_{l, k} \sum_{l^{\prime}, k^{\prime}} \frac{\partial^{2} \Phi}{\partial r_{i}(l, k) \partial r_{j}\left(l^{\prime}, k^{\prime}\right)}\right|_{0} u_{i}(l, k) u_{j}\left(l^{\prime}, k^{\prime}\right) \\
& + \text { h.o.t. }
\end{aligned}
$$

where h.o.t. stands for higher order terms, and for brevity the 0 subindex denotes a function evaluated at the equilibrium position.

The first-order term vanishes because it is evaluated at the equilibrium position:

$$
\left.\frac{\partial \Phi}{\partial r_{i}(l, k)}\right|_{0}=0
$$

Indeed, this term is the force exerted over each atom $(l, k)$ at equilibrium, which vanishes by construction $[54,55]$.

The total force acting on the individual atom $(l, k)$ in the $i$ direction is

$$
\begin{aligned}
F_{i}(l, k) & =-\frac{\partial V}{\partial r_{i}(l, k)} \\
& =-\left.\sum_{l^{\prime}, k^{\prime}} \frac{\partial^{2} \Phi}{\partial r_{i}(l, k) \partial r_{j}\left(l^{\prime}, k^{\prime}\right)}\right|_{0} u_{j}\left(l^{\prime}, k^{\prime}\right)+\text { h.o.t. }
\end{aligned}
$$

where repeated index denotes summation.

In the harmonic approximation, the higher-order terms are neglected under the assumption that the displacement of the atoms about their equilibrium position is small compared with the interatomic distances. Thus,

$$
\begin{aligned}
F_{i}(l, k) & =-\frac{\partial V}{\partial r_{i}(l, k)}=-\left.\sum_{l^{\prime}, k^{\prime}} \frac{\partial^{2} \Phi}{\partial r_{i}(l, k) \partial r_{j}\left(l^{\prime}, k^{\prime}\right)}\right|_{0} u_{j}\left(l^{\prime}, k^{\prime}\right) \\
& \equiv-\sum_{l^{\prime}, k} \Phi_{i j}\left(l, k ; l^{\prime}, k^{\prime}\right) u_{j}\left(l^{\prime}, k^{\prime}\right),
\end{aligned}
$$

where $\Phi_{i j}\left(l, k ; l^{\prime}, k^{\prime}\right) \equiv \boldsymbol{\Phi}$ is the Hessian operator of the interatomic potential, usually known as the force constant matrix (see $[53,55])$.

Owing to the translational symmetry of the crystalline lattice, we have [55]

$$
\Phi_{i j}\left(l, k ; l^{\prime}, k^{\prime}\right) \equiv \Phi_{j i}\left(l-l^{\prime}, k-k^{\prime}\right) \equiv \boldsymbol{\Phi}\left(l-l^{\prime}, k-k^{\prime}\right) .
$$

To wit, the force constant matrix depends only on the relative interatomic positions.

For a crystal in equilibrium, in the presence of some external force field $\boldsymbol{f}(l, k)$, the $(l, k)$ th atom will accordingly experience a displacement $\boldsymbol{u}(l, k)$ given by

$$
\boldsymbol{\Phi} \boldsymbol{u}(l, k)+\boldsymbol{f}(l, k)=0 .
$$

We must note that albeit this derivation applies on the harmonic lattice formalism, this does not mean that the interatomic potential, as given by Eq. (18), nor the force constant matrix $\boldsymbol{\Phi}$ are harmonic or linear.

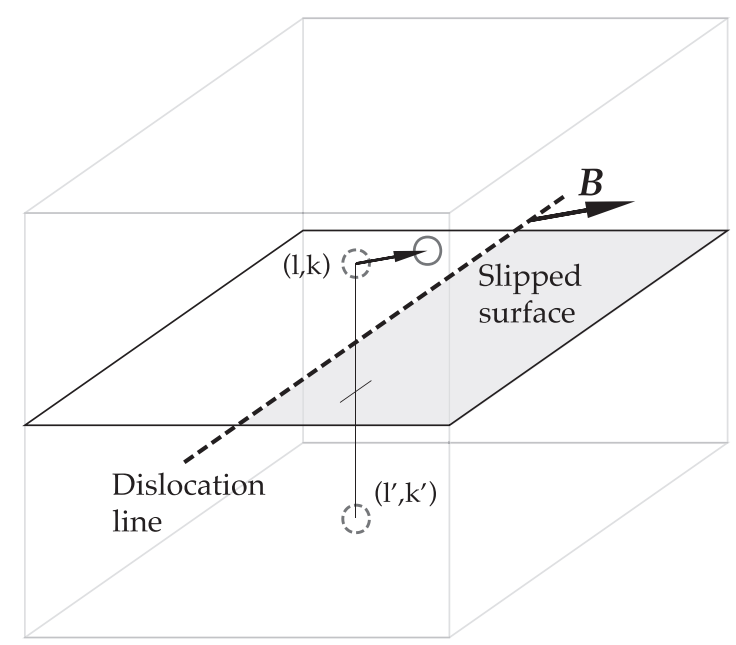

FIG. 3. The dislocation is defined by its line and the slip surface. The atoms at either side of the slip surface are displaced by a relative quantity $\boldsymbol{B}$, corresponding to the Burgers vector.

\section{B. Kanzaki forces of dislocations}

As in the continuum, dislocations are better understood in terms of the crystallographic disregistry they entail, rather than in terms of the forces necessary to produce the said disregistry. Still, the external force field $\boldsymbol{f}(l, k)$ necessary to generate the disregistry may be employed to define a dislocation in a perfect, defect-free crystalline lattice.

As is shown in Fig. 3, a dislocation is defined by its line, the slipped region, and the Burgers vector $\boldsymbol{B}$ [1]. Upon injection, the atoms at either side of the slip surface are displaced by a Burgers vector. In the following, we do not specify the alignment of $\boldsymbol{B}$ relative to the dislocation line, which for generality's sake in Fig. 3 we assume to be any. In addition, to avoid further complications, we define the slip surface in such a way that it does not intersect with any atom in the lattice.

Let us assume that, to begin with, the dislocation does not exist, and therefore that the crystal is at equilibrium. Then, $\boldsymbol{\Phi} \boldsymbol{u}(l, k)+\boldsymbol{f}(l, k)=0$ holds with $\boldsymbol{f}(l, k)=0$. At some stage, we introduce the dislocation by displacing the atoms across the slip surface by a Burgers vector, and then allow the lattice to relax itself back to equilibrium. Consider two atoms, $(l, k)$ and $\left(l^{\prime}, k^{\prime}\right)$, located at either side of the slip surface. The relative lattice positions of atoms $(l, k)$ and $\left(l^{\prime}, k^{\prime}\right)$ are given by $\boldsymbol{r}(l, k)-\boldsymbol{r}\left(l^{\prime}, k^{\prime}\right)$. Upon injecting the dislocation, the atoms will experience an additional relative displacement matching the disregistry imposed by the dislocation. Thus, after the dislocation's injection, the relative equilibrium position between the two atoms becomes $\boldsymbol{r}(l, k)-\boldsymbol{r}\left(l^{\prime}, k^{\prime}\right)-\boldsymbol{B}$.

In the final relaxed lattice, the equilibrium in the presence of the dislocation is still described by Eq. (23), and there would be no distinction between the forces involved in imposing the disregistry and those concerned with the subsequent lattice relaxation: the force on each atom would account for both effects. However, we may exploit Eq. (23) to describe the forces that one ought to apply on the perfect lattice to generate the disregistry. This relies on the superposition principle, which is enabled in the harmonic approximation owing to the 
linearity of Eq. (23), and to the fact that one assumes that the displacements off equilibrium are small.

Thus, we can describe the force acting on each atom as the sum of that due to the crystallographic slip across the cut surface $\boldsymbol{B}$, plus any additional displacements which would otherwise exist, and that are involved in the relaxation of the lattice. That is,

$$
\boldsymbol{f}^{\mathrm{tot}}(l, k)=\boldsymbol{f}^{\mathrm{disloc}}(l, k)+\boldsymbol{\Phi} \boldsymbol{u}(l, k),
$$

where $f^{\text {disloc }}$ is the part of the displacements produced by the atomic slip across the slip surface, up to the dislocation line. In light of Eq. (24), it is clear that knowledge of $\mathbf{u}$ provides immediate knowledge of the forces $f$ that acts as a source term for the said field $\mathbf{u}$ in the perfect lattice. Thus, as in the continuum [18], lattice force representations are uniquely determined by the displacement field. This is not necessarily the case for eigenstrains or other tensorial source representations, which cannot be uniquely determined from the displacement field $[18,19]$. As we do in the continuum, we do not concern ourselves with this displacement field's origin: we merely assume that the dislocation exists, and therefore accept that a force distribution $\boldsymbol{f}^{\text {disloc }}$ may be applied to enforce the disregistry.

The form of $f^{\text {disloc }}$ may be given. In direct analogy to the characteristic function used in Sec. II, let us define the following choice function (cf. [35]):

$$
\chi_{S}\left(l-l^{\prime}, k-k^{\prime}\right)= \begin{cases}+1, & \text { if }(l, k) \text { is above the slip surface and }\left(l^{\prime}, k^{\prime}\right) \text { below } \\ -1, & \text { if }(l, k) \text { is below the slip surface and }\left(l^{\prime}, k^{\prime}\right) \text { above } \\ 0, & \text { otherwise. }\end{cases}
$$

In the following, we omit discussing the dislocation core, which is dealt with in Sec. IV; here we are solely concerned with generating a Volterra dislocation in a lattice.

Thus, we can express the slip due to the dislocation as

$$
u_{j}^{\text {slip }}=\chi_{S}\left(l-l^{\prime}, k-k^{\prime}\right) B_{j} .
$$

The total force on atom $(l, k)$ is then

$$
f_{i}^{\mathrm{tot}}(l, k)=\sum_{l^{\prime}, k^{\prime}} \Phi_{i j}\left[u_{j}\left(l-l^{\prime}, k-k^{\prime}\right)-\chi_{S}\left(l-l^{\prime}, k-k^{\prime}\right) B_{j}\right],
$$

which must vanish at equilibrium. Thus, the additional force experienced by the atoms across the slip surface must be

$$
f_{i}^{\mathrm{disloc}}(l, k)=-\sum_{l^{\prime}, k^{\prime}} \Phi_{i j}\left(l-l^{\prime}, k-k^{\prime}\right) \chi_{S}\left(l-l^{\prime}, k-k^{\prime}\right) B_{j} .
$$

This equation provides the force distribution due to a dislocation in the harmonic lattice approximation; it is, therefore, the Kanzaki force of the Volterra dislocation. This definition of the Kanzaki force of a dislocation is analogous to that offered by Boyer and Hardy [33] and Caro and Glass [35], amongst many others. It represents the set of forces that would have to be applied on the perfect lattice to generate the topological displacements of a Volterra dislocation; it is, therefore, a distribution of Kanzaki forces. The force on an atomic row long after the dislocation core has passed is small again as the Kanzaki force acting on it is negated by the restoring force due to the harmonic lattice [38]. The total result of these two competing forces results in the required displacement jump of magnitude $B$ : the Kanzaki forces and the restoring forces from the harmonic lattice add to make the forces across the glide plane small again.

\section{Relationship between the Kanzaki and Burridge-Knopoff force representations}

The relationship between Eq. (28) and Eq. (6) may be established by considering the continuum "long-wave" limit of a harmonic lattice. Let us consider the case where the displacement generating the Kanzaki force is some general $u_{i}(l, k)$. The force in that case is

$$
\begin{aligned}
f_{i}(l, k) & =-\sum_{l^{\prime}, k^{\prime}} \Phi_{i j}\left(l-l^{\prime}, k-k^{\prime}\right) u_{j}\left(l^{\prime}, k^{\prime}\right) \\
& =\sum_{l^{\prime}, k^{\prime}} \Phi_{i j}\left(l-l^{\prime}, k-k^{\prime}\right) u_{j}\left(l-l^{\prime}, k-k^{\prime}\right),
\end{aligned}
$$

where $u_{j}\left(l-l^{\prime}, k-k^{\prime}\right)=u_{j}(l, k)-u_{j}\left(l^{\prime}, k^{\prime}\right)$, and where we have used

$$
\sum_{l^{\prime}, k^{\prime}} \Phi_{i j}\left(l-l^{\prime}, k-k^{\prime}\right) u_{j}(l, k)=0
$$

due to the homogeneity of the force constant matrix.

Now, let us extend by continuity the displacement $u_{j}(l, k)$, so that $u_{j}(l, k) \sim u_{j}(\boldsymbol{x})$, where $\boldsymbol{x} \in \mathbb{R}^{3}$ is now a continuous and sufficiently smooth ${ }^{1}$ variable such that $u_{j}(l, k)=u_{j}(\boldsymbol{x})$ when $\boldsymbol{x}=\boldsymbol{r}(l, k)$. This extension by continuity is justified when the characteristic wavelength of a displacement is larger than the interatomic spacing; hence, it is usually referred to as the long-wave approximation [54].

Equation (30) becomes

$$
f_{i}(\boldsymbol{x})=\sum_{l^{\prime}, k^{\prime}} \Phi_{i j}\left(l-l^{\prime}, k-k^{\prime}\right)\left[u_{j}(\boldsymbol{x})-u_{j}\left(\boldsymbol{x}^{\prime}\right)\right] .
$$

Expanding $u_{j}\left(\boldsymbol{x}^{\prime}\right)$ in Taylor series about $\boldsymbol{x}$ :

$$
\begin{aligned}
u_{j}\left(\boldsymbol{x}^{\prime}\right)-u_{j}(\boldsymbol{x})= & \left(x_{k}^{\prime}-x_{k}\right) \partial_{k} u_{j}(\boldsymbol{x}) \\
& -\frac{1}{2 !}\left(x_{k}^{\prime}-x_{k}\right)\left(x_{l}^{\prime}-x_{l}\right) \partial_{k} \partial_{l} u_{j}(\boldsymbol{x})+\text { h.o.t. }
\end{aligned}
$$

\footnotetext{
${ }^{1}$ For this approximation, it suffices that $u_{j} \in C^{2}\left(\mathbb{R}^{3}\right)$.
} 
If $u_{j}(\boldsymbol{x})$ varies by a small amount over the range of a $\left(l-l^{\prime}, k-k^{\prime}\right)$, we may truncate this expansion to second order. Upon substituting Eq. (32) into (31), we find

$$
\begin{aligned}
f_{i}(\boldsymbol{x}) \simeq & \sum_{l^{\prime}, k^{\prime}} \Phi_{i j}\left(l-l^{\prime}, k-k^{\prime}\right)\left(x_{k}-x_{k}^{\prime}\right) \partial_{k} u_{j}(\boldsymbol{x}) \\
& -\frac{1}{2 !} \sum_{l^{\prime}, k^{\prime}} \Phi_{i j}\left(l-l^{\prime}, k-k^{\prime}\right)\left(x_{k}-x_{k}^{\prime}\right) \\
& \times\left(x_{l}-x_{l}^{\prime}\right) \partial_{k} \partial_{l} u_{j}(\boldsymbol{x}) .
\end{aligned}
$$

The first term on the right-hand side vanishes because

$$
\begin{aligned}
& \sum_{l^{\prime}, k^{\prime}} \Phi_{i j}\left(l-l^{\prime}, k-k^{\prime}\right)\left(x_{k}-x_{k}^{\prime}\right) \partial_{k} u_{j}(\boldsymbol{x}) \\
& \quad=\partial_{k} u_{j}(\boldsymbol{x}) \sum_{l^{\prime}, k^{\prime}} \Phi_{i j}\left(l-l^{\prime}, k-k^{\prime}\right)\left(x_{k}-x_{k}^{\prime}\right) \\
& \quad=\partial_{k} u_{j}(\boldsymbol{x}) \sum_{l, k} \Phi_{i j}(l, k) x_{k}=0 .
\end{aligned}
$$

We are therefore left with

$$
f_{i}(\boldsymbol{x})=-\frac{1}{2 !} \partial_{l k}^{2} u_{j}(\boldsymbol{x}) \sum_{l^{\prime}, k^{\prime}} \Phi_{i j}\left(l-l^{\prime}, k-k^{\prime}\right)\left(x_{k}-x_{k}^{\prime}\right)\left(x_{l}-x_{l}^{\prime}\right) .
$$

The term in the sum defines the elastic constant tensor of a unit cell, which here we denote by $\bar{C}_{i j k l}$ (see [55]):

$$
\begin{aligned}
\bar{C}_{i j k l} & =-\frac{1}{2} \sum_{l^{\prime}, k^{\prime}} \Phi_{i j}\left(l-l^{\prime}, k-k^{\prime}\right)\left(x_{k}-x_{k}^{\prime}\right)\left(x_{l}-x_{l}^{\prime}\right) \\
& =-\frac{1}{2} \sum_{l, k} \Phi_{i j}(l, k) x_{k} x_{l} .
\end{aligned}
$$

If $V_{0}$ denotes the volume of a unit cell and $C_{i j k l}$ the elastic tensor, then, accounting for all symmetries [55],

$$
C_{i j k l}=\frac{1}{4 V_{0}}\left(\bar{C}_{i j k l}+\bar{C}_{j i l k}+\bar{C}_{i j l k}+\bar{C}_{j i k l}\right) .
$$

Thus, the force per atom in the unit cell is

$$
f_{i}(\boldsymbol{x})=\bar{C}_{i j k l} \partial_{l k}^{2} u_{j}(\boldsymbol{x}) .
$$

The force we are concerned about is the force per unit volume cell, for the force in Eq. (6) is a force per unit volume. The former may be obtained as [56]

$$
f_{i}(\boldsymbol{x})=\frac{1}{V_{0}} \int_{V^{\prime}} d \boldsymbol{x}^{\prime} \bar{C}_{i j k l} \partial_{l k}^{2} u_{j}\left(\boldsymbol{x}-\boldsymbol{x}^{\prime}\right) .
$$

Let us assume that $u_{j}\left(\boldsymbol{x}-\boldsymbol{x}^{\prime}\right)=B_{j} \chi_{S}\left(\boldsymbol{x}-\boldsymbol{x}^{\prime}\right)$ as expected for a Volterra dislocation. Then, we are concerned with

$$
f_{i}^{\text {disloc }}(\boldsymbol{x})=\frac{1}{V_{0}} \int_{V^{\prime}} d \boldsymbol{x}^{\prime} \bar{C}_{i j k l} \partial_{k} \partial_{l^{\prime}} B_{j} \chi_{S}\left(\boldsymbol{x}-\boldsymbol{x}^{\prime}\right) .
$$

Applying the divergence theorem to it

$$
f_{i}^{\text {disloc }}(\boldsymbol{x})=-C_{i k j l} B_{j} \partial_{k} \int_{S} \nu_{l} \chi_{S}\left(\boldsymbol{x}-\boldsymbol{x}^{\prime}\right) d S^{\prime} .
$$

We note that $\chi_{S}$ is integrated over unit-cell surfaces. The only instance in which it has a nonzero value is if the primitive cell lies along the slip surface, where the choice function changes value; in all other cells, the integral must vanish because the choice function does. Thus, the integral itself defines a characteristic function in the same way $\chi_{D}(\boldsymbol{x})$ did.

We can show this rigorously. For simplicity, we shall only concern ourselves with the case when $\boldsymbol{x}$ lies on top and $\boldsymbol{x}^{\prime}$ at the bottom (the opposite case being the converse of this one), so that $\chi_{S}\left(\boldsymbol{x}-\boldsymbol{x}^{\prime}\right)$ is a pure indicator function. This relates to the fact that the surface integral must also respect its own directionality. Let $D$ be the slip surface. Let $S$ be a unitcell surface. If $S \cap D$, then $\chi_{S} \cdot S=1 \equiv \mu_{D}(S \cap D)$, where here $\mu_{S \cap D}$ is in effect a Dirac measure over the intersection between the unit cell's surface and the slip surface, i.e.,

$$
\mu_{D}(S \cap D)= \begin{cases}1, & x \in S \cap D \\ 0, & x \notin S \cap D .\end{cases}
$$

Then, $\chi_{S} \cdot d S=d \mu_{D}$. We are merely seeking the integral of said measure over each and every unit cell that intersects the slip surface,

$$
\int_{S} d \mu_{D}=\mu_{D}(S \cap D) \equiv \chi_{D}(\boldsymbol{x}),
$$

which is by definition the characteristic function over the slip surface itself.

Thus, we conclude

$$
f_{i}^{\text {disloc }}(\boldsymbol{x})=-C_{i k j l} B_{j} \nu_{l} \partial_{k} \chi_{D}(\boldsymbol{x}) .
$$

This equation matches Eq. (6), by swapping indexes as follows:

$$
i \mapsto p, k \mapsto q, j \mapsto i, l \mapsto j
$$

and using the symmetry $C_{p q i j}=C_{i j p q}$. This proves that in the long-wave limit the Kanzaki forces of a Volterra dislocation in a lattice match the Burridge-Knopoff forces of a Volterra dislocation in the continuum.

Although the Kanzaki forces arise from the harmonic approximation of the interatomic energy landscape, they reflect the relaxed position of the atoms. With this proof, we show that, as with the Kanzaki forces of point defects, the Kanzaki forces of dislocations are nearly linear elastic, which justifies the use of the Kanzaki forces in an elastic continuum.

Equation (28) suggests that the force representation of a dislocation in a harmonic lattice extends beyond the atoms immediately above and below the slip surface, via the longrange pairwise interactions enforced by the force constant $\Phi_{i j}$, and because the choice function takes value \pm 1 across the slip surface, all atoms above and below the slip surface having been displaced by $\boldsymbol{B}$. That is, in principle, in order to generate the topology of the dislocations in perfect, defectfree crystalline lattice, all atoms above and below the nominal slip surface ought to have a Kanzaki force applied on them. However, given that these forces are relatively short range, they will only be noticeable in the environs of the slip surface and, in particular, at the dislocation core, where the imbalance is greatest because the atoms on the other side have not been displaced (further details on how to model the core are given in Sec. IV). As we have discussed in this section, in the continuum limit one assumes that these forces are short ranged (or, equivalently, that $\Phi_{i j}$ varies slowly across unit cells [56]). This means that in the continuum limit all the atoms for which 
the Kanzaki forces are large are subsumed under the slip surface, which in the continuum limit is infinitely thin.

Thus, although the Kanzaki forces of a dislocation in the harmonic lattice are of finite spatial width and applied along the slip surface, upon taking the continuum limit these forces are collapsed into the slip surface itself, leading to Eq. (44). As will be seen in Sec. IV, the Kanzaki forces are generally only large in the first layer immediately above and below the slip surface.

\section{KANZAKI FORCES OF DISLOCATIONS}

In Sec. III we have shown that, as with point defects, the Kanzaki forces of dislocations are also nearly elastic, and that the ensuing continuum level model comprises a set of Burridge-Knopoff forces distributed around the slip surface and the dislocation core. In this section, we discuss how one may employ the Kanzaki forces to build continuum models of the dislocation based on the Burridge-Knopoff forces.

The procedure is dual. We shall first establish a general methodology for computing the Kanzaki forces of a given dislocation. We then develop two different kinds of continuum models of the dislocation based on the Kanzaki-BurridgeKnopoff correspondence. To begin with, we may approximate the dislocation as a set of point forces applied on the equilibrium lattice positions, their magnitude being computed atomistically. This produces an elastic model of the dislocation core that accounts for short-range effects, and sheds light into the magnitude and directionality of core effects. Subsequently, we study and approximate the said effects via multipolar expansion [48] of the Kanzaki forces about the core.

\section{A. Computation of the force constant matrix in bec $\mathbf{W}$}

The Kanzaki forces on a dislocation are given by Eq. (24). For a Volterra dislocation, we have

$$
f_{i}^{\text {disloc }}(l, k)=-\sum_{l^{\prime}, k^{\prime}} \Phi_{i j}\left(l-l^{\prime}, k-k^{\prime}\right) \chi_{S}\left(l-l^{\prime}, k-k^{\prime}\right) B_{j} .
$$

Given that dislocations are extended defects, applying singlepoint calculations to compute them as done in point defects [31] appears less straightforward than the explicit calculation of the force constant matrix $\Phi_{i j}$ for a given interatomic force field and the subsequent computation of the Kanzaki forces via Eq. (28). The $\Phi_{i j}$ force constant matrix may be obtained from first principles $[57,58]$ or, if available, from phenomenological interatomic potentials such as those available for molecular dynamics simulations [59].

The accuracy of the phenomenological potential (or of the $a b$ initio calculation) will impact $\Phi_{i j}$ and the calculations we describe in the following. We note, however, that the Kanzaki forces are defined as harmonic forces. Thus, in computing the $\Phi_{i j}$ matrix within the harmonic approximation we are not introducing further methodological errors to those already made in their definition.

Here, we compute the force constant matrix from the embedded atom interatomic potential $[60,61]$ of bcc tungsten developed by Marinica et al. [62]. A lattice statics code was written and employed in a simple central difference method

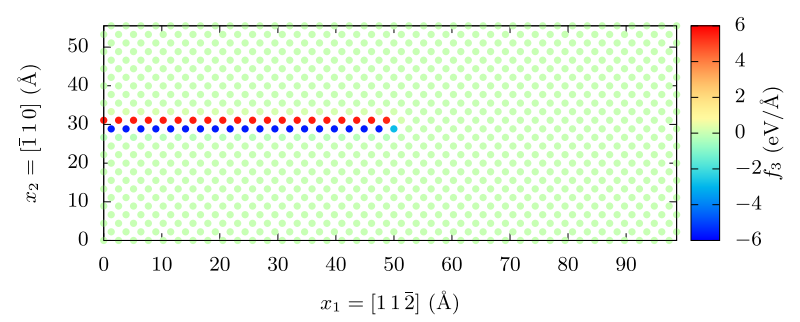

(a) Cut across $(\overline{1} 10)$.

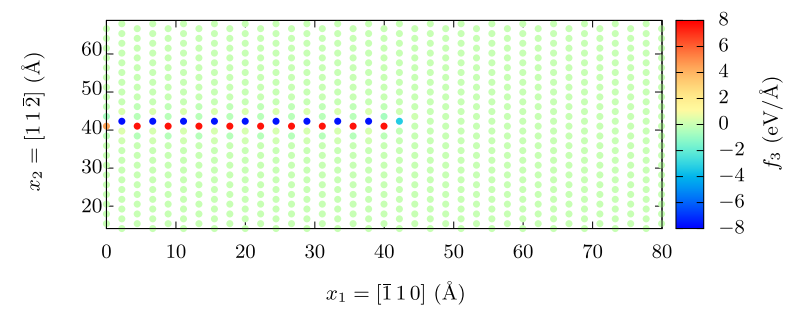

(b) Cut across $(11 \overline{2})$.

FIG. 4. Magnitude of the $f_{3}$ the slip Kanzaki forces across the cut surface for a $1 / 2\left\langle\begin{array}{lll}1 & 1 & 1\end{array}\right\rangle$ screw dislocation in bcc W.

with step size $h$ to approximate the force constant matrix as

$$
\Phi_{i j}=\frac{f_{j}\left(x_{i}-h\right)-f_{j}\left(x_{i}+h\right)}{2 h},
$$

where the indices $i, j$ run over all particles and dimensions. Here, $f_{j}$ is the force induced on atom $j$ upon displacing atom $i$ along direction $x_{i}$ by a magnitude $\pm h$, as indicated (see [63]). Further details on the computation of the dynamic force matrix may be found in the Supplemental Material [65].

\section{B. Computation of the Kanzaki forces of a screw dislocation in bec $\mathbf{W}$}

\section{Kanzaki forces associated with the Volterra dislocation}

The topology of the Volterra dislocation may be reproduced via the $\chi_{S}\left(l-l^{\prime}, k-k^{\prime}\right) B_{j}$ Volterra displacement, which is then used in combination with the $\Phi_{i j}$ matrix calculated with Eq. (46) to compute $f_{i}^{\text {disloc }}(l, k)$, i.e., the dislocation's Kanzaki forces using Eq. (28).

Here, we primarily consider a $1 / 2\langle 111\rangle$ screw disloca-

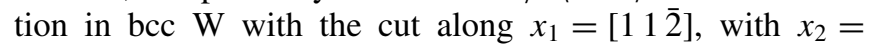
[1 10$], x_{3}=\left[\begin{array}{lll}1 & 1 & 1\end{array}\right]$, as shown in Fig. 4(b). The displacements generating the Volterra dislocation in this case are $u_{3}=B \mathrm{H}\left(-x_{1}\right) \delta\left(x_{2}\right)$. Using this displacement and the force constant matrix computed for bcc W in Eq. (28), we obtain the Kanzaki forces represented in Fig. 4. Away from the core, we observe that along the first atomic row there act two forces of equal magnitude but opposite sign; as is shown in Fig. 4, the average force at either side of the cut surface is $\pm 5.602 \mathrm{eV} / \AA$ in the first layer immediately above and below the cut surface, and $\mp 0.22 \mathrm{eV} / \AA$ along the second layer. In the subsequent atomic layers, these forces have vanished.

The orientation of the cut is irrelevant in generating the Volterra dislocation. Indeed, if the cut is placed along the $x_{2}=\left[\begin{array}{lll}1 & 1 & 0\end{array}\right]$ direction instead [see Fig. 4(a)], the magnitude of the slip Kanzaki forces is $\pm 7.636 \mathrm{eV} / \AA$ along the first layer, and $\mp 0.24 \mathrm{eV} / \AA$ along the second layer. The differences in magnitudes are due to the different interatomic spacing 
between the atoms at either side of the cut; however, as is discussed below, the net distributed force is the same irrespective of where the cut is placed; this confirms the wellknown feature of the Volterra dislocation that the orientation of the cut surface is irrelevant in generating the dislocation. Thus, the Kanzaki forces across the cut surface appear to be extremely short range, and heavily localized at either side of the slip surface, in a way already heavily reminiscent of the Burridge-Knopoff forces.

\section{Kanzaki forces associated with the dislocation core}

Given that no further provisions were made for the core, this calculation does not represent its geometry adequately; in particular, the assumption that it can be modeled as a Volterra core via the $\chi_{S}\left(l-l^{\prime}, k-k^{\prime}\right) B_{j}$ displacements is deemed too coarse. We may refine the dislocation's Kanzaki force field by first obtaining the actual equilibrium core structure, and then computing the Kanzaki forces necessary to generate the core's topology. We note that due to the linear nature of the Kanzaki forces, the core Kanzaki forces, understood as those pertaining the non-Volterra topology of the core, can be computed separately from the slip Kanzaki forces, which we define as those related to the disregistry across the cut surface. The core Kanzaki forces may therefore be computed by (a) subtracting the $B_{j} \chi_{S}\left(l-l^{\prime}, k^{\prime} k^{\prime}\right)$ displacement from the relaxed dislocation structure, and computing the Kanzaki forces; or (b) substracting the slip Kanzaki forces from the Kanzaki forces obtained from the global equilibrium configuration. Both sets of Kanzaki forces can then be superimposed to generate the final topology of the dislocation, which will consist of slip and core displacements. We note that these Kanzaki forces are obtained from the dislocated topology of the crystal, which is prescribed by the interatomic force field. The Kanzaki forces do not uniquely define the energetic pathway from the perfect lattice to the dislocation, nor is this path necessarily reversible: they describe the final, relaxed configuration of the dislocated lattice, whatever this might be.

Here, we first determine the equilibrium core configuration by minimizing a $1 / 2\langle 111\rangle$ straight screw dislocation in bcc W using LAMMPS [64], an MD software package, and the same bcc W interatomic potential as above [62]. We follow the usual core minimization procedure detailed in [10], by first introducing the dislocation by imposing the Volterra displacements along the antiplane direction in a $100 \times 200 \times 12$ periodic unit box for the same crystallographic orientation as before; the box is large enough to ensure that we avoid interatomic self-interactions in all directions, and that the dislocation's periodic images are sufficiently far away not to substantially affect the core. We then minimize the system, from where we obtain the dislocation's core displacements as predicted by the interatomic potential. Using Eq. (28) for the core's minimized displacements, we finally compute the core Kanzaki force components as shown in Fig. 5. As can be seen, the core Kanzaki forces consist of in-plane $f_{1}$ and $f_{2}$ components, the magnitude of which is about an order of magnitude smaller than that of the slip Kanzaki forces, i.e., $\mathrm{O}(0.1) \mathrm{eV}$ vs $\mathrm{O}(1) \mathrm{eV}$. This is because the core's differential displacements are equally about an order of magnitude smaller than the actual Burgers vector. The core's degeneracy,

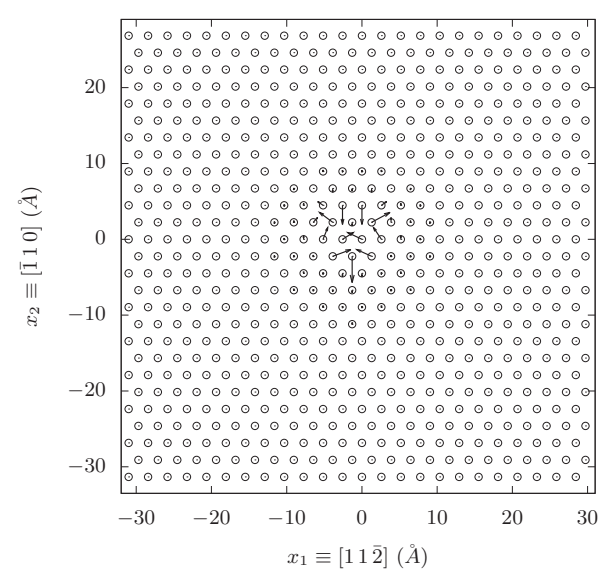

FIG. 5. $\left(f_{1}, f_{2}\right)$ core Kanzaki force components about the core. Note that the forces are scaled so that 5 units of length represents $1 \mathrm{eV} / \AA$.

as predicted by the Marinica interatomic potential employed here, is reflected in the symmetry of the distribution of core Kanzaki forces.

\section{ATOMISTICALLY INFORMED ELASTIC MODELS OF THE DISLOCATION AND THE DISLOCATION CORE}

The dislocation, understood as a Kanzaki force distribution, may therefore be represented as the superposition of the slip Kanzaki forces (shown in Fig. 4) and the core Kanzaki forces (shown in Fig. 5). Having established in Sec. III B the correspondence between the Kanzaki and the BurridgeKnopoff forces, here we argue that the dislocation may be represented in a linear elastic continuum by considering each and every Kanzaki force that defines it.

The general procedure to build such model is the following. Having computed the slip Kanzaki forces and their point of application, we define the following distribution of point Kanzaki forces:

$$
f_{p}(\boldsymbol{x})=\sum_{n} f_{p}^{n} \delta\left(\boldsymbol{x}-\boldsymbol{x}^{n}\right),
$$

where $f_{p}^{n}$ is each Kanzaki force applied on $\boldsymbol{x}^{n}$, as computed in the atomistic model. The corresponding linear elastic field field is then given by

$$
u_{i}(\boldsymbol{x})=\sum_{n} f_{p}^{n} G_{i p}\left(\boldsymbol{x}-\boldsymbol{x}^{n}\right),
$$

where $G_{i p}(\boldsymbol{x})$ is the elastic Green's function (see the Supplemental Material [65] for a detailed derivation of the elastic fields). Given that bcc tungsten is heavily isotropic, in the following we shall concern ourselves with the isotropic Green's function alone. The field is singular at the point of application of each force, that is, at each equilibrium atomic position. Given that the extension by elastic continuation of an inherently atomistic field is meaningless at such short-range distance from the atomic position, such singularity appears physically irrelevant in the current model, albeit it is liable to regularization, if so needed, via nonlocal extensions to linear elasticity (see, for instance, [66,67]). 


\section{Modeling of the Volterra dislocation using the slip Kanzaki forces}

Away from the atomic positions, the field given by Eq. (48) generates the dislocation. If we distinguish between the core and the slip Kanzaki forces, we may identify the atomistic Volterra dislocation as that generated by the slip Kanzaki forces alone:

$$
u_{i}(\boldsymbol{x})^{\text {Volterra }}=\sum_{n} f_{K} G_{i 3}\left(\boldsymbol{x}-\boldsymbol{x}^{n}\right) .
$$

In the case of the screw dislocation in tungsten, $f_{K}=$ $\pm 7.636 \mathrm{eV} / \AA$ right above and below the slip surface along [ 1110$]$, and $f_{K}= \pm 5.60248 \mathrm{eV} / \AA$ right above and below the slip surface along [ $\left[\begin{array}{lll}1 & 1 & \overline{2}\end{array}\right]$.

The slip Kanzaki forces are clearly liable to homogenization as a distribution of force doublets applied over the slip surface, leading to their Burridge-Knopoff form. We first express the distribution of slip Kanzaki forces as $f_{K}(\boldsymbol{x})=$ $\sum_{n} f_{K} \chi_{S}\left(\boldsymbol{x}-\boldsymbol{x}^{n}\right)=f_{K} \sum_{n} \chi_{S}\left(x_{1}-n \Delta x, x_{2}\right)$ where $\Delta_{x}$ is the spacing between atoms along the $x_{1}$ direction, and taking the limit $n \rightarrow 0$, we find

$$
f_{3}^{\text {slip }}(\boldsymbol{x})=\frac{f_{K}}{\Delta x} \delta^{\prime}\left(x_{2}\right) \mathrm{H}\left(x_{1}\right) .
$$

We may therefore model the slip Kanzaki forces as a uniform distribution of force doublets of magnitude $f_{K} / \Delta x$. For the screw dislocation in $\mathrm{W}, f_{K}=5.602 \mathrm{eV} / \AA, \Delta x=a / \sqrt{6}=$ $1.29 \AA$ if the cut is along [ $\left[\begin{array}{lll}1 & 1 & 2\end{array}\right]$, and $f_{K}=7.636 \mathrm{eV} / \AA, \Delta x=$ $a / \sqrt{2}=2.22 \AA$ if the cut is along $\left[\begin{array}{lll}1 & 1 & 0\end{array}\right]$. Either of these values corresponds with $45.16 \mathrm{~N} / \mathrm{m}$, which is to an excellent approximation the magnitude of the distributed BurridgeKnopoff dipoles given by Eq. (8), i.e., $B \mu=45.08 \mathrm{~N} / \mathrm{m}$ for the $\mu=163 \mathrm{GPa}, B=2.7492 \AA$ predicted by the interatomic potential; that is, irrespective of the orientation of the cut surface, we find that $\frac{f_{K}}{\Delta x}=B \mu$. Thus, we directly obtain the Burridge-Knopoff representation of the Volterra dislocation given by Eq. (8) from the slip Kanzaki forces.

We conclude that the slip Kanzaki forces are equivalent to the source terms employed in the continuum force representation of a Volterra dislocation.

\section{Modeling the dislocation core using the core Kanzaki forces}

The core Kanzaki forces may be employed to model the short-range fields of a dislocation core using linear elasticity, without recourse of higher-order local theories of elasticity. ${ }^{2}$ In the present calculations, we find two reasons for this. The first one is that the core Kanzaki forces are about 10 times smaller than the slip Kanzaki forces: if the slip Kanzaki forces are commonly employed, as we have already shown, to model the Volterra dislocation using linear elasticity, then the weaker core Kanzaki forces may as well be employed within the remit of linear elasticity to generate a model of the core. The second reason is that the magnitude of the in-plane displacements leading to the core Kanzaki forces will at most be of about

\footnotetext{
${ }^{2}$ This refers to higher-order expansions of the strain tensor, leading to nonlinear theories of elasticity that approximate better the dispersion relation of the harmonic lattice [16].
}

the same magnitude as the slip across the cut surface, which clearly allows them to be treated under the small-displacement approximation and therefore linear elasticity. Still, nonlocal and gradient corrections (cf. [66,68,69]) could be introduced to regularize the elastic field at the atomic positions.

If we take the core Kanzaki forces alone, then the core's own displacement fields will be given by the representation theorem

$$
u_{i}(\boldsymbol{x})^{\mathrm{core}}=\sum_{n} f_{p}^{n, \text { core }} G_{i p}\left(\boldsymbol{x} \boldsymbol{x}^{n}\right) .
$$

These core fields, a more detailed expression of which may be found in the Supplemental Material [65], can be superimposed to those due to the Volterra dislocation.

We note that the core Kanzaki forces consist of two inplane $f_{1}$ and $f_{2}$ components (see Fig. 5). This entails that the in-plane displacement field components $u_{1}$ and $u_{2}$ are nonzero and, accordingly, that there exist certain in-plane elastic field components due to the core of the dislocation which is neither predicted nor expected in the Volterra description of a screw dislocation, since the latter is obtained from antiplane considerations alone, as are the slip Kanzaki forces. The presence of these in-plane core fields appears nonetheless necessary in view of the often reported in-plane core displacements [2] associated with the nonplanarity of the core [3], given that as we have shown here the in-plane core displacements will inevitably entail in-plane core Kanzaki forces. In the current context, these in-plane fields have been noted in the past [70-73], and even prompted suggestions for the need of a three-dimensional model of the core [73]. Furthermore, their presence can be attested: in the MD minimization we performed here to attain the core structure, we observe the presence of the hydrostatic in-plane field shown in Fig. 6(a), which again cannot be associated to a Volterra screw dislocation, but to the in-plane core displacements.

Here, we focus on the hydrostatic stress component because the Volterra screw dislocation only accounts for deviatoric stresses: the hydrostatic field about the core serves as an illustration of the additional core effects that the Kanzaki force formalism allows to capture and model in the elastic continuum. Employing Eq. (51) for the core Kanzaki forces in Fig. 5, we generate an elastic model of the screw dislocation core in bcc W (see the Supplemental Material [65] for further details on their derivation using the isotropic Green's function). Figure 6(b) depicts the magnitude of the resulting hydrostatic stress field component of the core in the elastic continuum. As can be seen, the shape and magnitude of the elastic model's core fields compare very well to those of the atomistic calculation [see Fig. 6(a)]. This proves that the methodology proposed here may be employed to model the dislocation core. We note that the core's field along the $x_{1}=0$ direction is technically regularized: no singularities may be found along the epicentral line because no atoms lie on it. This is significant because in dislocations dynamics simulations dislocations only experience short-range interactions with one another along the slip $x_{1}$ direction [10,74]. However, the atoms about the cut lying on either side of the cut surface are close enough to the latter that the influence of their own elastic fields is vast as $x_{1}$ approaches one of them; in any case, so are the atomistic stress fields in such short ranges. Otherwise, 

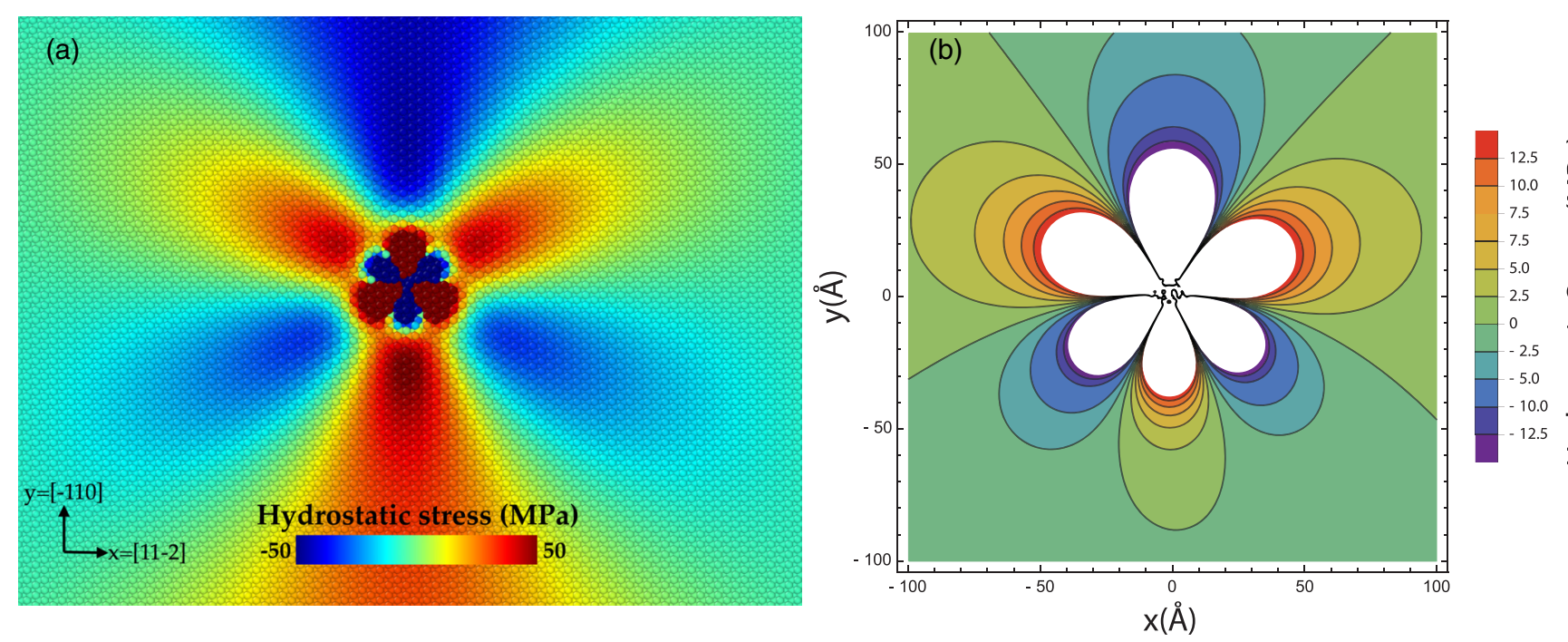

FIG. 6. The presence of a weak $1 / r^{2}$ decaying hydrostatic stress field in (a) is justified by the presence of in-plane core Kanzaki forces necessary to generate the core's topology. We find that a linear elasticity model of the core employing those Kanzaki forces, shown in (b), adequately captures the main characteristics of the core, including its magnitude and rate of decay.

what this approach shows is that the core Kanzaki forces may be employed to model within the continuum the short-range fields due to core effects that can be readily measured in atomistic simulations of dislocations.

Depending on the number of core Kanzaki forces involved, the modeling of the core via all its core Kanzaki forces may quickly become protracted, all the more because as can be seen in Fig. 6, the magnitude of the core's in-plane field is small and, as will be shown in Sec. VI, it decays with $1 / r^{2}$ in the far field. In the following section, we detail how to produce simpler corrections to the core's fields based on the multipolar field expansion of the Kanzaki force distribution.

\section{MULTIPOLAR MOMENTS OF DISLOCATIONS}

The description of dislocations as a set of Kanzaki (or Burridge-Knopoff) forces enables the definition of the multipolar moment expansion associated with said forces. Multipolar field expansions are commonly employed in the modeling of point defects [16,17], whereby the point defect's elastic field is modeled in terms of the multipole moments $[18,19]$ implied by the Kanzaki forces defining the defect. The multipole moments substitute the defect for point force dipoles, quadrupoles, octopoles, ... acting on the center of the defect. Because the elastic fields of these multipolar arrangements decay with increasing rate, even the lower, dipole field accurately models the long-range field of the defect $[48,75]$.

Multipolar field expansions may be provided for any force distribution; details on the multipolar field expansions of the Burridge-Knopoff continuum dislocations may be found in [48]. Here, we are concerned with computing the multipolar moments implied by the Kanzaki forces.

In general, the $n$ th-order multipolar moment of a given force distribution is given by [48]

$$
\gamma_{p k_{1} \ldots k_{n}}^{(n)}=\int_{\Omega} x_{k_{1}} \cdot \ldots \cdot x_{k_{n}} f_{p}(\boldsymbol{x}) d \Omega
$$

where $f_{p}(\boldsymbol{x})$ are the Kanzaki forces described above. Given they are a discrete set of forces applied over specific equilibrium positions which we label as $\mathbf{r}(l, k)$, we may express the multipolar moment as (see [17])

$$
\gamma_{p k_{1} \ldots k_{n}}^{(n)}=\sum_{l, k} r_{k_{1}} \cdot \ldots \cdot r_{k_{n}} \cdot f_{p}(l, k) .
$$

\section{A. Multipolar moments of the slip Kanzaki forces}

Neither the integral in Eq. (52) nor the sum in Eq. (53) are guaranteed to converge for single dislocations. For instance, if $f_{3}=B \mu \mathrm{H}\left(-x_{1}\right) \delta^{\prime}\left(x_{2}\right)$ represents the individual screw dislocation, then

$$
\begin{aligned}
\gamma_{32}^{(1)} & =\int_{\mathbb{R}^{2}} x_{2} B \mu \mathrm{H}\left(-x_{1}\right) \delta^{\prime}\left(x_{2}\right) d x_{1} d x_{2} \\
& =B \mu \int_{0}^{\infty} \mathrm{H}\left(-x_{1}\right) d x_{1}=\infty .
\end{aligned}
$$

This is because sums over the Kanzaki and Burridge-Knopoff forces along their direction of application require the force distributions have finite, compact support. This means that individual dislocations do not have a well-defined multipolar moment. In general, we can say that the $n$ th-order multipolar moments of a dislocation are well defined and finite only if the slip surface of the dislocation is finite. This means that only dislocation loops or clusters of straight dislocations (dipoles, quadrupoles, ...) have well-defined multipolar moments.

A proof similar to the one given in Sec. II B 1 concerning the resultant $f_{y}$ component in edge dislocations may be given. In the continuous case, $f_{p}(\boldsymbol{x})=-C_{i j p q} B_{i} v_{j} \partial_{q} \chi_{D}(\boldsymbol{x})$, whereupon (cf. [48])

$$
\begin{aligned}
\gamma_{p k_{1} \ldots k_{n}}^{(n)}= & -C_{i j p q} B_{i} v_{j}\left(\sum_{i=1}^{n} \delta_{k_{i} q} \prod_{j=1, j \neq i}^{n} \delta_{k_{j} u_{\pi_{j j i}}}\right) \\
& \times \int_{\Omega} x_{u_{1}} \cdot \ldots \cdot x_{u_{n-1}} \chi_{D}(\boldsymbol{x}) d \Omega
\end{aligned}
$$


with

$$
\pi_{j ; i}= \begin{cases}j, & j<i \\ j-1, & j>i .\end{cases}
$$

Given that $x_{u_{1}} \cdot \ldots \cdot x_{u_{n-1}}$ are polynomials, the integrand in Eq. (55) is continuous and bounded over the support of $\chi_{D}(\boldsymbol{x})$, i.e., compactly supported over $D$. Thus, the integral in Eq. (55) is guaranteed to exist if and only if $D$ is finite. If $\chi_{D}(\boldsymbol{x})$ represents an individual straight dislocation, $D$ is not bounded: it takes values over a half-line in $\mathbb{R}$. Thus, the multipolar moments of individual dislocations are not generally defined.

The discrete (atomistic) case is a particular case of the latter, where the Kanzaki force on the atom $(l, k)$ is of the form

$$
f_{p}=-\sum_{l, k} \Phi_{p q} B_{q} \chi_{S}\left(l, k ; l^{\prime}, k^{\prime}\right)
$$

across the cut surface. A detailed proof applied to the lattice model is given in the Supplemental Material [65].

The physical interpretation of this result is as follows. The multipolar moments are the sum of the products of atomic positions and their corresponding, local, Kanzaki force components. If the topology of the defect is finite, as is the case with point defects, with the dislocation core (see Sec. VI), or if the slip surface is finite, they will have a finite value (zero or otherwise). If the slip surface is infinite, then the summation is performed over infinite terms of increasing value (as the value of $\mathbf{r}$ increases) and of opposite sign across the slip surface. This series, in discrete or integral form, is not guaranteed to converge unless it is a finite sum. The series is in fact Cèsaro summable, but generally divergent. In either case, one or more of the multipolar moments will diverge, rendering them ill defined.

The inability to define the multipolar moments of straight dislocations is therefore inherent to the slip Kanzaki forces and the Burridge-Knopoff forces of a Volterra dislocation. However, we may define the multipolar moments associated with clusters of straight dislocations such as dislocation dipoles.

For illustrative purposes, here we consider a dipole of screw dislocations in bcc W, separated by some distance $L$. We may compute then the value of $\gamma_{p k_{1}}^{(1)}$ for a number of dipoles of increasing size by first computing their respective slip Kanzaki forces in a manner analogous to what we have described; as we show in Fig. 7, beyond dipoles of very small size, we are able to find a linear increase in the dipolar moment with the dipole length. Thus, we may define a mean multipolar moment

$$
\bar{\gamma}_{p k_{1} \ldots k_{n}}^{\text {slip }}=\frac{1}{L^{n}} \sum_{l, k} r_{k_{1}} \cdot \ldots \cdot r_{k_{n}} \cdot f_{p}(l, k) .
$$

In our calculations, $\bar{\gamma}_{23}^{\text {slip }}=3.75 \mathrm{eV} / \AA$ (or $2.75 \mathrm{eV} / \AA^{2}$ per unit Burgers). The dipolar moment predicted by the BurridgeKnopoff continuum force representation is $\frac{\gamma_{23}^{\mathrm{B}}}{B L}=\mu$; thus, $B \mu=2.749 \mathrm{eV} / \AA^{2}$ agrees with the atomistic dipolar moment.

This slip dipole moment is less important than the core dipole moment we define in the following. This is because the

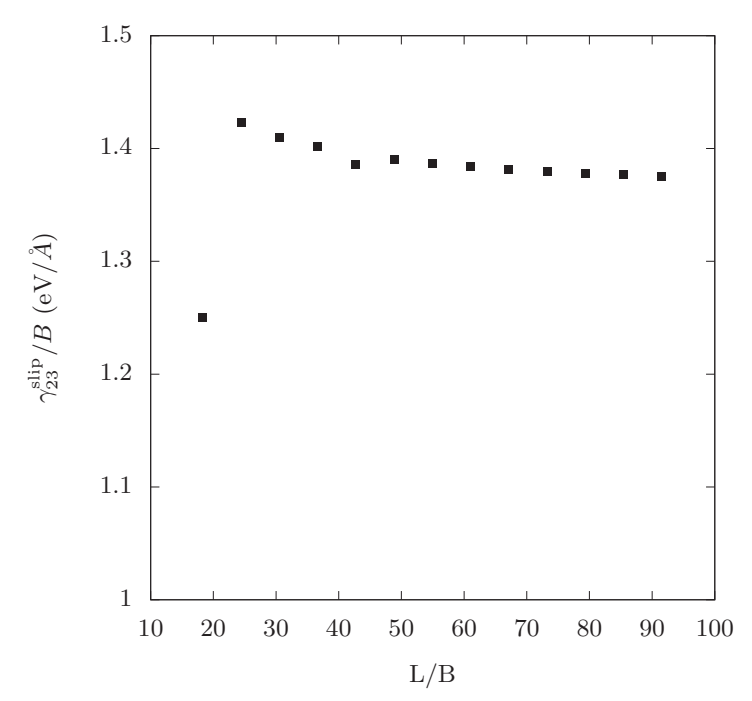

FIG. 7. Computed values of $\gamma_{23}^{\text {slip }}$ for a dipole of screw dislocations in bcc W for different dipole separation lengths.

dipole (or higher-order multipole) fields of the slip surface, i.e., those pertaining to the Volterra dislocation's topology, are only accurate in capturing the long-range fields of the dislocation, and are generally expected to be accurate at distances greater than about 5-10 the length of a dipole [48]. In the context of atomistic calculations, these are vast distances; however, the atomistic computation of the slip dipole or higher-order multipole moments might be useful for estimating the deviation between the long-range "isotropic" dipole fields and the anisotropic ones, which the slip dipole moments computed from the slip Kanzaki forces would naturally compute.

\section{B. Multipolar moments of the dislocation core}

As was argued in Sec. V A2, the core Kanzaki forces on their own may be employed as source terms in the elastic continuum to refine the Volterra dislocation core. Owing to the potentially large number of forces necessary to do this, such model may be protracted to evaluate. However, applying the force multipole field expansion methodology just discussed, we may develop a much simpler model of the dislocation's core. In doing so, we are effectively subsuming the Kanzaki forces into force multipoles acting on the geometrical site of the core.

The Volterra dislocation's core, be it as described by its classical Burridge-Knopoff forces or using the slip Kanzaki forces, does not entail multipolar moments at the core, since the core has no spatial width. However, the core Kanzaki forces we have computed here do because they arise from in-plane displacements. Thus, invoking the BK-Kanzaki force correspondence, we can assign a multipolar moment to the core, in addition to those associated with the dislocation and the cut surface. Unlike the latter, however, the core's multipolar moments are well defined irrespective of the support of the cut surface because the dislocation core itself is localized, rather than extended. Otherwise, the computation of the $n$ th-order core multipolar moment follows Eq. (53), with $f_{p}$ in this case the core Kanzaki forces. 

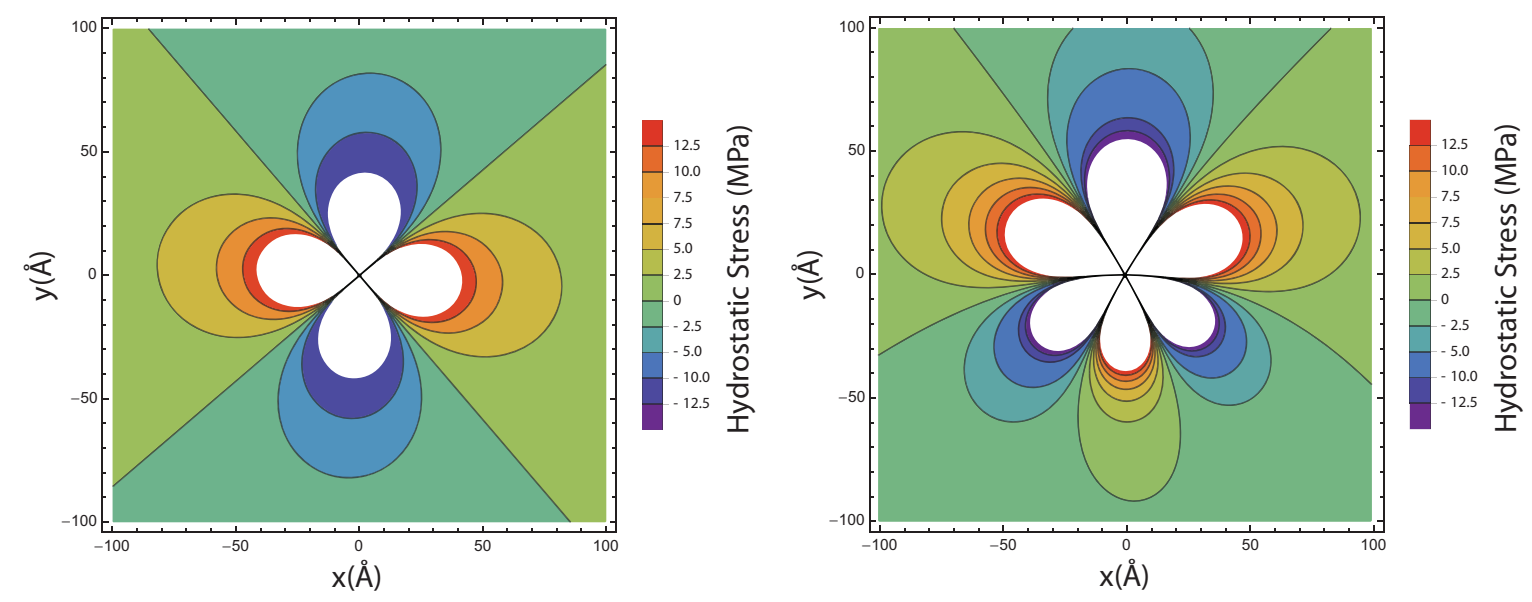

FIG. 8. Dipolar and quadrupolar order core corrections of the screw dislocation core.

In the case of the screw dislocation in bcc $\mathrm{W}$, we are particularly interested in the dipolar and quadrupolar moments. This is because of the threefold symmetry of the core: if we are to subsume it into a force dipole, this symmetry is lost, and the dipolar approximation will, as is discussed below, lead to relatively inaccurate symmetrization of the field in the short range. This is solved by adding the second-order, quadrupolar terms, which lead to a highly accurate correction of the core.

For the screw dislocation in bcc $\mathrm{W}$ under consideration, the in-plane dipolar moments are

$$
\begin{array}{ll}
\gamma_{11}^{\text {core }}=-1.766 \mathrm{eV}, & \gamma_{12}^{\text {core }}=0.507 \mathrm{eV}, \\
\gamma_{21}^{\text {core }}=-0.654 \mathrm{eV}, & \gamma_{22}^{\text {core }}=-2.708 \mathrm{eV},
\end{array}
$$

and the in-plane quadrupolar moments are

$$
\begin{aligned}
& \gamma_{111}^{\text {core }}=10.237 \mathrm{eV} \AA, \quad \gamma_{121}^{\text {core }}=\gamma_{211}^{\text {core }}=15.446 \mathrm{eV} \AA, \\
& \gamma_{222}^{\text {core }}=-23.743 \mathrm{eV} \AA, \gamma_{221}^{\text {core }}=\gamma_{221}^{\text {core }}=1.135 \mathrm{eV} \AA, \\
& \gamma_{112}^{\text {core }}=31.3 \mathrm{eV} \AA, \gamma_{122}^{\text {core }}=\gamma_{212}^{\text {core }}=3.497 \mathrm{eV} \AA .
\end{aligned}
$$

Thus, we may build a correction to the core of the Volterra dislocation by superimposing the slip Kanzaki forces or the Burridge-Knopoff source term associated with the slip across the cut surface with the dipolar or quadrupolar core fields. The Burridge-Knopoff forces associated with the slip are

$$
f_{3}=B \mu \mathrm{H}\left(x_{1}\right) \delta^{\prime}\left(x_{2}\right), \quad f_{1}=0=f_{2} .
$$

This force generates the Volterra dislocation's fields (see Sec. II B 1 and the Supplemental Material [65]), associated to which we now define the dipolar and quadrupolar force corrections, which modifies the values of $f_{1}$ and $f_{2}$ into either (cf. [48])

$$
\begin{aligned}
& f_{1}=\gamma_{11}^{\text {core }} \delta^{\prime}\left(x_{1}\right)+\gamma_{12}^{\text {core }} \delta^{\prime}\left(x_{2}\right), \\
& f_{2}=\gamma_{21}^{\text {core }} \delta^{\prime}\left(x_{1}\right)+\gamma_{22}^{\text {core }} \delta^{\prime}\left(x_{2}\right)
\end{aligned}
$$

for the dipolar approximation, or

$$
f_{p}=f_{p}^{\text {dipolar }}+\gamma_{p k_{1} k_{2}}^{\text {core }} \partial_{k_{1} k_{2}} \mathrm{H}\left(x_{1}\right) \delta\left(x_{2}\right), \quad p, k_{1}, k_{2}=1,2
$$

for the quadrupolar approximation.
These additional in-plane $f_{1}$ and $f_{2}$ forces generate the multipolar core correction, the elastic fields of which may be obtained using the multipolar moments above in combination with the elastic Green's function. In general, we have that the multipolar fields are of the form [48]

$$
u_{i}(\boldsymbol{x})=\sum_{n=0}^{\infty} \frac{(-1)^{n}}{n !} \frac{\partial^{n} G_{i p}(\boldsymbol{x})}{\partial x_{k_{1}} \ldots \partial x_{k_{n}}} \gamma_{p k_{1} \ldots k_{n}}^{(n)}
$$

for $n$ the multipolar expansion's order. The dipolar core correction is then

$$
u_{i}^{(1)}(\boldsymbol{x})=\left[\frac{\partial G_{i p}(\boldsymbol{x})}{\partial x_{k_{1}}}\right] \gamma_{p k_{1}}^{\text {core }}
$$

and the quadrupolar correction

$$
u_{i}^{(2)}(\boldsymbol{x})=\left[\frac{\partial G_{i p}(\boldsymbol{x})}{\partial x_{k_{1}}}\right] \gamma_{p k_{1}}^{\text {core }}-\frac{1}{2}\left[\frac{\partial^{2} G_{i p}(\boldsymbol{x})}{\partial x_{k_{1}} \partial x_{k_{2}}}\right] \gamma_{p k_{1} k_{2}}^{\text {core }} .
$$

The associated stress fields can be obtained from differentiation of the displacement fields, and are reproduced in the Supplemental Material [65] due to their length.

Figure 8 shows the hydrostatic field due to the dipolar and quadrupolar core corrections of the $1 / 2\langle 111\rangle$ screw dislocation in bcc $\mathrm{W}$; this figure might be compared to the "exact" linear elastic model of the core, accounting for all Kanzaki force components given in Fig. 6. As may be seen in Fig. 8(a), albeit the dipole field captures the long-range fields adequately, it fails to reproduce correctly the symmetry of the shorter-range fields owing to the difficulties in representing an object with threefold symmetry employing force dipoles alone. At shorter ranges, the second-order quadrupolar field, shown in Fig. 8(b), is seen to offer far greater accuracy. This is because the addition of force quadrupoles in the three directions of space, along with the original force dipoles of the dipolar approximation, combine to recover the core's threefold symmetry.

Due to the nature of the elastic Green's function and its derivatives (see the Supplemental Material [65]), the stress field associated with the core decays as $1 / r^{n+1}$ for the $n$th multipolar moment expansion term $[15,17,48]$. Given that the dipole field is the lowest-order nonzero multipolar component of the elastic field of the core Kanzaki forces, we conclude that 
the stress field of the dislocation core decays with $1 / r^{2}$ in the far field, and that its magnitude is controlled by the first-order dipolar moments of the dislocation core.

The quadrupolar terms, in turn, decay with $1 / r^{3}$. We may provide an estimate of the accuracy of the dipolar core correction based on the spatial range where the quadrupolar terms overtake in value those due to the dipolar correction. The correction therefore remains valid until

$$
r_{\text {low }}^{(1)} \approx\left|\frac{\gamma_{p k_{1} k_{2}}^{\text {core }}}{\gamma_{p k_{1}}^{\text {core }}}\right| \text {. }
$$

For our present calculations, $r_{\text {low }}^{(1)}=2-30 B$, depending on the direction of the field. A comparison between Figs. 8(a) and 8 (b) suggests this estimate is adequate: the magnitude of both fields about $30 B \approx 60 \AA$ away from the core is very similar; the extent of the far field agrees well with the core field estimates obtained by Henager et al. [70,71] to dipolar order using energetic considerations. The same argument may be applied to establish the lower bound of the quadrupolar approximation, by computing the octopolar moments (not reproduced here, but the expansion of which decays with $\left.1 / r^{4}\right)$. In our calculations, that sets the range of accuracy of the quadrupolar expansion at $r_{\text {low }}^{(2)} \approx 0.8-5 B$, i.e., within the usual bounds of the dislocation core [1].

The dipolar and quadrupolar core corrections reveal a number of features of the dislocation core. On the one hand, as we have shown any core effect associated with local in-plane (or, arguably, antiplane) non-Volterra atomic displacements will decay in the far field with $1 / r^{2}$, and the field's magnitude will that of the core's own dipolar moments. In the case of the screw dislocation in $\mathrm{W}$ under consideration here, this entails the presence of a weak in-plane hydrostatic stress field (and, correspondingly, of a weak in-plane deviatoric field we have not reproduced here). This effect is separate from the one that may be obtained from regularizing the Volterra dislocation's core by, for instance, employing a PeierlsNabarro (PN) model. As with the PN model, this in-plane effect concerns non-Volterra core displacements, but these are found in the in-plane direction, rather than in the antiplane one, and are entirely due to the actual structure of the dislocation core.

On the other hand, we note that the range over which the core fields apply extends well beyond the usual range that short-range regularizations do. For example, the short-range Peierls-Nabarro regularization of the core tends to apply for about $10 B$ about the core [1], and further away the model's elastic fields match those of the Volterra dislocation. In this case, however, and as can be seen in Fig. 8, the core correction extends further away (about $50 \mathrm{~B}$ in the case of bcc W). Unlike the Peierls-Nabarro model [76,77] or Foreman's corrections [78], our core Kanzaki model of the core is an exact mapping of the actual core structure: the core Kanzaki forces are spatially extended to all atomic positions making up the relaxed core structure. The magnitude of the core Kanzaki forces is a true representation of the source term generating the atomic core displacement. At the same time, we must stress that unlike the PN model, the elastic model of the core we propose here does not regularize it: it does not introduce any constructive hypothesis that would cap or render the stress at the dislocation line zero but, rather, takes the atomistically predicted core Kanzaki forces as source terms to generate the dislocation core in an elastic continuum. What we show here is not how to regularize the core, but rather how to better capture its short-range fields based solely on atomistic Kanzaki forces, leading to a truly three-dimensional model of the core. Still, the elastic core models proposed here could be regularized by employing gradient elasticity corrections (see for instance [67]).

\section{DISCUSSION AND CONCLUSIONS}

This paper has discussed the intimate relation between the continuum description of dislocations as distributed BurridgeKnopoff forces and the atomistic treatment of dislocations as Kanzaki forces. Both the Burridge-Knopoff and the Kanzaki forces are applied over a defect-free system, and both act as source terms in their respective continuum and atomistic formalisms; neither represent explicitly applied forces but, rather, the forces one ought to apply in the perfect continuum or in the perfect crystalline lattice in order to generate the topology of a dislocation.

We have explored in detail how crystalline dislocations are represented as force distributions in the continuum invoking the Burridge-Knopoff representation theorem [20]. We have provided the general form of the Burridge-Knopoff force distributions of both closed loops and straight dislocations, highlighting a number of physical limitations to the existence of the latter. In exploring the connection between the continuum Burridge-Knopoff and the atomistic Kanzaki forces, we have rigorously shown that in the long-wave limit, the Kanzaki forces of a general dislocation loop converge to the Burridge-Knopoff forces of a Volterra dislocation loop, thereby establishing a rigorous mathematical connection between the two.

This facilitates the use of the atomistic Kanzaki forces, or a homogenization of the latter, as source terms to generate dislocations in the continuum, thereby leading to a number of viable models to bridge the gap between the atomistic nature of dislocations, captured by the Kanzaki forces, and their continuum long-range study, captured by the BurridgeKnopoff forces.

In order to showcase this, we have discussed how the Kanzaki forces of dislocations may be calculated in atomistic models, via the computation of the material's lattice force constant matrix. In our calculations, we have focused on bcc $\mathrm{W}$, and have employed a well-known interatomic potential [62] to compute the force constant matrix; without loss of generality for the methodology presented here, more complex or accurate methods such as DFT could be employed to compute the said matrix.

With the force constant matrix of bcc $\mathrm{W}$ in place, we have computed the Kanzaki forces of a straight 1/2 1111$\rangle$ screw dislocation. We have shown that the Kanzaki forces of a dislocation may be superimposed to account for two effects, one related to the slip surface, which leads to the "slip Kanzaki forces," and another related to the dislocation core, which leads to the "core Kanzaki forces."

The slip Kanzaki forces arise from the need to impose a disregistry along the slip surface; this disregistry, of mag- 
nitude $B$, entails the presence of a distribution of Kanzaki forces along the surface where it is applied (i.e., along the slip surface). We have seen that the presence of the slip Kanzaki force is a requirement for the topological closure of the dislocation, namely, that individual dislocations need the presence of a companion dislocation of opposite sign in the crystal, for otherwise the force distribution becomes unnormalizable. This confirmation of Frank's rule for the conservation of mass highlights a fundamental distinction between dislocations understood as crystallographic defects (which is the sole concern of this work), and dislocations understood as kernels of strain in generalized continua (which may allow for mass not to be conserved). Furthermore, we have shown that the slip Kanzaki forces match with great accuracy the Burridge-Knopoff forces that generate a Volterra dislocation.

In turn, the core Kanzaki forces are related to the particular topology of the dislocation core, which entails the presence of an additional set of Kanzaki forces heavily localized about the core. These core Kanzaki forces do not have a direct correspondence in the continuum model of a Volterra dislocation nor any of the usual continuum core regularizations (see [1]), which concern the slip across the cut surface. Here, we have calculated the core Kanzaki forces by first minimizing the core structure of the dislocation and computing the atomic displacements necessary to generate the topology of the core, and then combining these core displacements with the force constant matrix of the perfect crystal, resulting in the core Kanzaki forces proper.

The core Kanzaki forces may be employed, as per the Burridge-Knopoff and Kanzaki force correspondence, as source forces in the elastic continuum to better model the dislocation core. In the case of the screw dislocation studied here, the core forces have components in the dislocation plane, as opposed to the slip forces acting solely in the antiplane direction. Thus, they entail in-plane hydrostatic and deviatoric stresses which are not present in the Volterra model of a screw dislocation.

These two field components may be modeled in a number of ways. As the most direct approximation within the limits of linear elasticity, the individual core Kanzaki forces may be employed to generate the whole core, which would be superposed to the Burridge-Knopoff forces generating the Volterra displacement. Alternatively, here we have also shown how the Kanzaki forces (and the Burridge-Knopoff) forces may be employed to generate a multipolar field expansion of their implied elastic fields. In doing so, we subsume the Kanzaki (or Burridge-Knopoff) force distribution into a force dipole, quadrupole, octopole,.... The multipolar field expansions of the slip Kanzaki forces are of interest in the study of the long-range fields of clusters of dislocations or dislocation loops, but their accuracy depends on the dimensionality of the cluster (i.e., the separation between dislocations in a dipole, or the area-perimeter ratio in a dislocation loop) [48]. However, the multipolar field expansions of the core Kanzaki forces are far more accurate because the core itself is local and not extended.

Using the multipolar expansion of the core Kanzaki force, we have shown that the core's own stress fields decay with $1 / r^{2}$, so that their additional in-plane stress fields are relevant only in short-range interactions. Furthermore, we have also noted that, at least in the case of the screw dislocation in bcc W, the magnitude of the core Kanzaki forces is about an order of magnitude weaker than that of the slip Kanzaki forces, which entails that these core elastic fields not only decay faster than the dislocation's, but are generally an order of magnitude weaker. Arguably as well, given that the core Kanzaki forces are weaker than the slip Kanzaki forces, the need for higher-order (i.e., nonlinear) models of elasticity to treat the core seems unjustified to model the short-range effects of the core away from the atomic positions themselves: the displacements at the core are not so large that such models would be warranted, so linear elasticity, or gradient models of linear elasticity, would appear sufficient.

The core corrections proposed here do not regularize the core, in the sense that they do not limit the magnitude of the stress at the core; that may be achieved if a nonlocal model were to be employed (see for instance [66]). On the contrary, the multipolar core corrects the dislocation core's fields beyond the planarity implied by the Volterra dislocation's core, which in turn underpins prior attempts at correcting the dislocation core, including the Peierls-Nabarro model or core models relying on gradient elasticity. None of these cases contemplate a three-dimensional core that includes a shortrange hydrostatic field about the core of a screw dislocation.

Thus, by showing the equivalence between the continuum Burridge-Knopoff force representation and the atomistic Kanzaki forces of dislocations, we have been able to establish a methodology for the development of atomistically informed elastic models of a dislocation. The procedure entails the following:

(1) Compute the lattice's force constant matrix.

(2) Compute the slip Kanzaki forces imposing a Volterra displacement across the slip surface.

(3) Minimize the core structure, and obtain the core's topological displacements.

(4) Compute the core Kanzaki forces, imposing the core's topological displacements.

(5) Build the elastic model of the dislocation using the slip Kanzaki forces and the core Kanzaki forces.

(6) Approximate the core via the dipolar or quadrupolar fields of the core Kanzaki forces.

We note that the Kanzaki forces serve to describe the final, dislocated topology, whatever that might be, but not the pathway to inject the dislocation in the lattice. They are not generally reversible either because the reference state of the path reverting the dislocation back to the perfect lattice is the dislocated lattice itself, and not the perfect lattice used to define the dislocation's Kanzaki forces.

The computation of the atomic displacements about the core is commonplace in the literature, particularly in the study of the fundamental causes of slip in bcc metals, where the nonplanar nature of the dislocation core is often regarded as crucial in explaining the onset of slip in bcc metals [2-4,25]. This often leads to the presentation of differential displacement maps [79], representing the predicted position of the atoms at the core relative to their perfect lattice positions [3]. This paper shows that said atomistic modeling efforts can be extended to the elastic continuum by employing the differential displacements of the core atoms to compute their 
corresponding core (and slip) Kanzaki forces. This can be readily achieved, as we show in the paper, by computing the force constant matrix of the system, which is accessible both to $a b$ initio calculations and to molecular dynamics calculations employing empirical interatomic potentials. Invoking the equivalence between the Kanzaki and Burridge-Knopoff force representations we have established here, the core Kanzaki forces enable the production truly three-dimensional models of the core in the elastic continuum of considerable accuracy, which account for physically motivated effects that most nonVolterra core representations miss.

The incorporation of the Kanzaki force based core fields in continuum models will be relevant in a varied number of applications. For instance, by altering the short-range interactions between screw dislocations, it is possible they will influence locking and pileup mechanisms. These short-range core corrections affecting the near field about the core over extended length scales (about $\approx 100 \mathrm{~B}$ ) will also be of relevance where interactions between dislocations and point defects is relevant. This includes studies of solute strengthening (see for instance $[24,46]$ ) where the Kanzaki force model of the dislocation core may be employed alongside the Kanzaki force model of the solute in the matrix to model their respective interactions more accurately. The same core Kanzaki forces may also improve diffusion models of atomic species such as hydrogen about the dislocation core via the additional hydrostatic pressure field $[47,80,81]$. Other applications in this area would include the study of dislocation-precipitate interactions in the near field [45]. Additionally, the Kanzaki force representation of a dislocation may prove valuable in the bridging between atomistic and continuum length scales in dislocation dynamics (cf. [44]), as it may provide a better representation of the dislocation's near field in the overlap region between the atomistic and continuum level models. In all those cases, our model offers a straightforward way of accounting for short-range effects in a physically motivated fashion.

\section{ACKNOWLEDGMENTS}

B.G.-L. is thankful to the Master and Fellows of Trinity College Cambridge for the financial support under the author's Title A fellowship. J.V. was supported through a studentship in the Centre for Doctoral Training on Theory and Simulation of Materials at Imperial College funded by EPSRC under Grant No. EP/L015579/1.

B.G.-L. conceived the continuum mathematical models and wrote the paper. J.V. conceived and performed the atomistic calculations. Both authors contributed to the writing of the discussion. The authors report no competing interests. This work does not have any experimental data. All simulation results are made available upon request to the corresponding author.
[1] J. P. Hirth and J. Lothe, Theory of Dislocations, 2nd ed. (Wiley, New York, 1982).

[2] K. Ito and V. Vitek, Philos. Mag. A 45, 1387 (2001).

[3] V. Vitek, Prog. Mater. Sci. 36, 1 (1992).

[4] J. A. Moriarty, V. Vitek, V. V. Bulatov, and S. Yip, J. Comput, Aided Mater. Des. 9, 99 (2002).

[5] D. L. Olmsted, L. G. Hector, W. A. Curtin, and R. J. Clifton, Modell. Simul. Mater. Sci. Eng. 13, 371 (2005).

[6] S. Queyreau, J. Marian, M. R. Gilbert, and B. D. Wirth, Phys. Rev. B 84, 064106 (2011).

[7] U. F. Kocks, A. S. Argon, and M. F. Ashby, Thermodynamics and Kinetics of Slip (Pergamon, Oxford, 1975), Chap. 1, pp. 1-281.

[8] G. Regazzoni, U. F. Kocks, and P. S. Follansbee, Acta Metall. 35, 2865 (1987).

[9] B. Gurrutxaga-Lerma, D. S. Balint, D. Dini, and A. P. Sutton, J. Mech. Phys. Solids 84, 273 (2015).

[10] V. V. Bulatov and W. Cai, Computer Simulations of Dislocation (Oxford University Press, Oxford, UK, 2006).

[11] I. Groma, F. F. Csikor, and M. Zaiser, Acta Mater. 51, 1271 (2003).

[12] R. Hill, The Mathematical Theory of Plasticity, Vol. 11 (Oxford University Press, Oxford, 1998).

[13] X. Markenscoff, J. Elast. 10, 193 (1980).

[14] J. D. Eshelby, Proc. R. Soc. A 252, 561 (1957).

[15] T. Mura, Micromechics of Defects in Solids, 2nd ed. (Kluwer, Amsterdam, 1982).

[16] C. Teodosiu, Elastic Models of Crystal Defects (Springer, London, 1982).

[17] R. W. Balluffi, Introduction To Elasticity Theory For Crystal Defects, 2nd ed. (World Scientific, Singapore, 2017).
[18] G. Backus and M. Mulcahy, Geophys. J. Int. 46, 341 (1976).

[19] G. Backus and M. Mulcahy, Geophys. J. Int. 47, 301 (1976).

[20] R. Burridge and L. Knopoff, Bull. Seismol. Soc. America 54, 1875 (1964).

[21] F. A. Dahlen, Geophys. J. Int. 28, 357 (1972).

[22] V. V. Bulatov, F. F. Abraham, L. P. Kubin, B. Devincre, and S. Yip, Nature (London) 391, 669 (1998).

[23] S. Ando, H. Tonda, and T. Gotoh, Metall. Mater. Trans. A 33, 823 (2002).

[24] S. I. Rao, C. Varvenne, C. Woodward, T. A. Parthasarathy, D. Miracle, O. N. Senkov, and W. A. Curtin, Acta Mater. 125, 311 (2017).

[25] S. L. Frederiksen and K. W. Jacobsen, Philos. Mag. 83, 365 (2003).

[26] C. Woodward, D. R. Trinkle, L. G. Hector Jr, and D. L. Olmsted, Phys. Rev. Lett. 100, 045507 (2008).

[27] M. Ghazisaeidi and D. R. Trinkle, Acta Mater. 60, 1287 (2012).

[28] H. Kanzaki, J. Phys. Chem. Solids 2, 24 (1957).

[29] H. R. Schober and K. W. Ingle, J. Phys. F: Met. Phys. 10, 575 (1980).

[30] C. Domain and C. S. Becquart, Phys. Rev. B 65, 024103 (2001).

[31] R. Nazarov, J. S. Majevadia, M. Patel, M. R. Wenman, D. S. Balint, J. Neugebauer, and A. P. Sutton, Phys. Rev. B 94, 241112 (2016).

[32] A. A. Maradudin, J. Phys. Chem. Solids 9, 1 (1959).

[33] L. L. Boyer and J. R. Hardy, Philos. Mag. 24, 647 (1971).

[34] N. E. Glass, J. Phys. (Paris) 44, 741 (1983).

[35] J. A. Caro and N. Glass, J. Phys. (Paris) 45, 1337 (1984).

[36] V. Celli and N. Flytzanis, J. Appl. Phys. 41, 4443 (1970).

[37] I. A. Kunin, Mechanics of Generalized Continua (Springer, New York, 1968), pp. 321-329. 
[38] S. Ishioka, J. Phys. Soc. Jpn. 30, 323 (1971).

[39] T. Ninomiya, J. Phys. Soc. Jpn. 25, 830 (1968).

[40] T. Mura, in Continuum Models of Discrete Systems, edited by J. W. Provan and H. H. E. Leipholz (University of Waterloo Press, Waterloo, Canada, 1977), pp. 503-519.

[41] S. R. de Debiaggi and A. Caro, J. Phys. (Paris) 48, 1499 (1987).

[42] Wang Shaofeng, Phys. Rev. B 65, 094111 (2002).

[43] S. Haq, A. B. Movchan, and G. Rodin, J. Appl. Mech. 74, 686 (2007).

[44] G. Anciaux, T. Junge, M. Hodapp, J. Cho, J.-F. Molinari, and W. A. Curtin, J. Mech. Phys. Solids 118, 152 (2018).

[45] A. Takahashi and N. M. Ghoniem, J. Mech. Phys. Solids 56, 1534 (2008).

[46] G. P. M. Leyson, L. G. Hector Jr, and W. A. Curtin, Acta Mater. 60, 3873 (2012).

[47] Y. Gu and J. A. El-Awady, J. Mech. Phys. Solids 112, 491 (2018).

[48] B. Gurrutxaga-Lerma, Int. J. Eng. Sci. 128, 165 (2018).

[49] W. Rudin, Principles of Mathematical Analysis, 3rd ed. (McGraw-Hill, New York, 1976).

[50] R.-J. Lange, J. High Energy Phys. 11 (2012) 032.

[51] F. R. N. Nabarro, Theory of Crystal Dislocations (Oxford University Press, Oxford, 1967).

[52] U. F. Kocks and R. O. Scattergood, Acta Metall. 17, 1161 (1969).

[53] A. A. Maradudin, E. W. Montroll, and G. H. Weiss, in Theory of Lattice Dynamics in the Harmonic Approximation, edited by F. Seitz and D. Turnbull, Solid State Physics Vol. 3 (Academic, New York, 1963).

[54] M. Born and K. Huang, Dynamical Theory of Crystal Lattices (Oxford University Press, New York, 1998).

[55] N. W. Ashcroft and N. D. Mermin, Solid State Physics (Brooks/Cole, Belmont, MA, 1976).

[56] E. Kröner and B. K. Datta, in Fundamental Aspects of Dislocation Theory, Conference Proceedings, Vol. 2, edited by J. A. Simmons, R. deWit, and R. Bullough, Institute of Materials Research (National Bureau of Standards, Washington D.C., 1969), pp. 737-746.

[57] G. J. Ackland, M. C. Warren, and S. J. Clark, J. Phys.: Condens. Matter 9, 7861 (1997).

[58] S. Baroni, S. De Gironcoli, A. Dal Corso, and P. Giannozzi, Rev. Mod. Phys. 73, 515 (2001).
[59] E. Hayward, C. Deo, B. P. Uberuaga, and C. N. Tomé, Philos. Mag. 92, 2759 (2012).

[60] M. S. Daw and M. I. Baskes, Phys. Rev. B 29, 6443 (1984).

[61] M. W. Finnis and J. E. Sinclair, Philos. Mag. A 50, 45 (1984).

[62] M.-C. Marinica, L. Ventelon, M. R. Gilbert, L. Proville, S. L. Dudarev, J. Marian, G. Bencteux, and F. Willaime, J. Phys.: Condens. Matter 25, 395502 (2013).

[63] M. Finnis, Interatomic Forces in Condensed Matter, edited by A. P. Sutton and R. E. Rudd, Oxford Series on Materials Modelling Vol. 1 (Oxford University Press, Oxford, UK, 2003).

[64] S. Plimpton, P. Crozier, and A. Thompson, LAMMPS-largescale atomic/molecular massively parallel simulator, Technical Report, Sandia National Laboratories, 2007.

[65] See Supplemental Material at http://link.aps.org/supplemental/ 10.1103/PhysRevB.98.134104 for a detailed derivation of the elastic fields of a dislocation using force representations, an expression of the dipolar and quadrupolar core corrections, and a discussion of the divergence of the multipolar moments of individual dislocations in lattice model.

[66] R. D. Mindlin, Arch. Ration. Mech. Anal. 16, 51 (1964).

[67] M. Lazar, Philos. Mag. 97, 3246 (2017).

[68] A. C. Eringen, J. Phys. D: Appl. Phys. 10, 671 (1977).

[69] R. C. Picu, J. Mech. Phys. Solids 50, 1923 (2002).

[70] C. H. Henager and R. G. Hoagland, Scr. Mater. 50, 1091 (2004).

[71] C. H. Henager and R. G. Hoagland, Philos. Mag. 85, 4477 (2005).

[72] H. Kimizuka and S. Ogata, Phys. Rev. B 84, 024116 (2011).

[73] J. Verschueren, B. Gurrutxaga-Lerma, D. S. Balint, D. Dini, and A. P. Sutton, J. Mech. Phys. Solids 98, 366 (2017).

[74] E. Van der Giessen and A. Needleman, Modell. Simul. Mater. Sci. Eng. 3, 689 (1995).

[75] R. W. Balluffi, S. M. Allen, and W. C. Carter, Kinetics of Materials (Wiley, Hoboken, NJ, 2005).

[76] F. R. N. Nabarro, Proc. Phys. Soc. 59, 256 (1947).

[77] R. Peierls, Proc. Phys. Soc. 52, 34 (1940).

[78] A. J. Foreman, M. A. Jaswon, and J. K. Wood, Proc. Phys. Soc. A 64, 156 (1951).

[79] M. S. Duesbery, V. Vitek, and D. K. Bowen, Proc. R. Soc. London A 332, 85 (1973).

[80] H. Yu, A. Cocks, and E. Tarleton, J. Mech. Phys. Solids (2018), doi: 10.1016/j.jmps.2018.08.020.

[81] I. H. Katzarov, D. L. Pashov, and A. T. Paxton, Phys. Rev. Mater. 1, 033602 (2017). 